TRANSACTIONS OF THE

AMERICAN MATHEMATICAL SOCIETY

Volume 362, Number 6, June 2010, Pages 2963-299

S $0002-9947(10) 04903-2$

Article electronically published on January 21, 2010

\title{
LINEAR PROGRESS IN THE COMPLEX OF CURVES
}

\author{
JOSEPH MAHER
}

\begin{abstract}
We show that a random walk on the mapping class group of an orientable surface of finite type makes linear progress in the relative metric, which is quasi-isometric to the complex of curves.
\end{abstract}

\section{Contents}

1. Introduction 2963

2. Preliminaries 2967

3. Halfspaces 2969

4. Non-elementary semi-groups 2980

5. Exponential decay 2983

6. Linear progress 2986

Acknowledgements

\begin{tabular}{ll} 
References & 2989 \\
\hline
\end{tabular}

\section{INTRODUCTION}

Let $\Sigma$ be an orientable surface of finite type, which is not a sphere with three or fewer punctures. The mapping class group $G$ of $\Sigma$ is the group of orientationpreserving diffeomorphisms of $\Sigma$, modulo those isotopic to the identity. Let $\mu$ be a probability distribution on $G$. We may use $\mu$ to generate a random walk on $G$, which is a Markov chain on $G$ with transition probabilities $p(x, y)=\mu\left(x^{-1} y\right)$, and we will assume we start at the identity at time zero. The path space for the random walk is the probability space $\left(G^{\mathbb{Z}_{+}}, \mathbb{P}\right)$, where the product $G^{\mathbb{Z}_{+}}$is the collection of all sample paths, and the measure $\mathbb{P}$ is determined by $\mu$. Let $w_{n}$ be the random variable corresponding to projection onto the $n$-th factor. So if $\omega$ is a sample path, then $w_{n}(\omega)$ is the location of the path at time $n$, and the distribution of $w_{n}$ is given by the $n$-fold convolution of $\mu$ with itself, which we shall write $\mu_{n}$. We say that the random walk has a linear rate of escape if the limit $\ell=\lim _{n \rightarrow \infty} \frac{1}{n}\left|w_{n}(\omega)\right|$ exists for almost all sample paths $\omega$, and $\ell$ is strictly greater than zero almost surely. Here $|g|$ is the length of the group element $g$ in the word metric on the group $G$. Kesten [18, 19] and Day [4 showed that an irreducible random walk on a non-amenable group has a linear rate of escape, assuming the random walk has finite first moment, i.e. the expected value of the distance the random walk moves in one step is finite. The mapping class group contains non-abelian free subgroups, so in particular is

Received by the editors February 8, 2008.

2000 Mathematics Subject Classification. Primary 37E30, 20F65, 60J10. 
non-amenable. Therefore a random walk on the mapping class group makes linear progress in the word metric on the group.

Masur and Minsky [27] show that there is a relative metric on the mapping class group, under which the group is quasi-isometric to the complex of curves. A relative metric is a word metric on the group with respect to an infinite generating set, consisting of a finite generating set, union a finite collection of subgroups. In this case, the collection of subgroups consists of stabilizers of simple closed curves $\alpha_{i}$, where the $\alpha_{i}$ consist of representatives for orbits of simple closed curves under the action of the mapping class group. The complex of curves is a simplicial complex whose vertices are isotopy classes of essential simple closed curves, and whose simplices are spanned by disjoint collections of simple closed curves. The quasi-isometry may be explicitly described by choosing a basepoint $x_{0}$ for the complex of curves, and sending $g$ to $g\left(x_{0}\right)$. We will write $\widehat{d}(1, g)$ for the length of the group element $g$ in the relative metric on $G$, and this is coarsely equivalent to the distance that $g$ moves the basepoint in the complex of curves. In this paper we show that a random walk in the mapping class group makes linear progress in the relative metric.

Theorem 1.1. Let $G$ be the mapping class group of an orientable surface of finite type, which is not a sphere with three or fewer punctures, and consider the random walk generated by a probability distribution $\mu$, whose support is bounded in the relative metric, which generates a non-elementary subgroup of the mapping class group, and which has finite first moment. Then there is a constant $\ell>0$ such that $\lim _{n \rightarrow \infty} \frac{1}{n} \widehat{d}\left(1, w_{n}\right)=\ell$ almost surely.

The relative metric is an improper metric on the mapping class group, and therefore is not quasi-isometric to the word metric, so linear progress in the word metric does not immediately imply linear progress in the relative metric. We will begin with a brief discussion of the mapping class group of the torus, as in this case it is easy to see that the result holds.

The mapping class group of the torus is isomorphic to the group $S L(2, \mathbb{Z})$. As there are no non-parallel disjoint essential simple closed curves on the torus, the curve complex for the torus is usually defined to have edges connecting pairs of curves that intersect exactly once. The curve complex for the torus is the Farey triangulation of the unit disc, and $S L(2, \mathbb{Z})$ with its word metric is quasi-isometric to the dual graph, which is a trivalent tree. This is illustrated in Figure 1 .

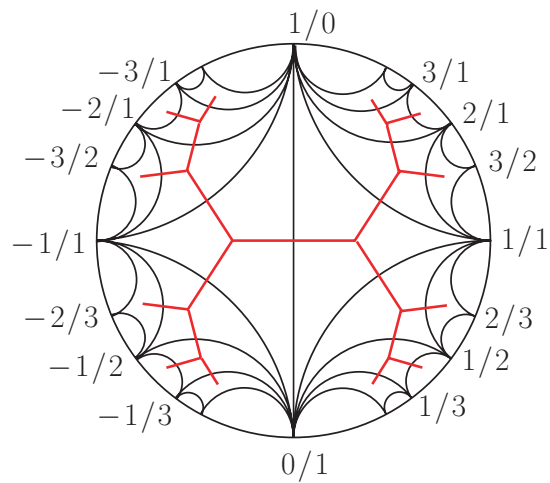

Figure 1. The Farey triangulation. 
For simplicity, consider a non-backtracking random walk on the trivalent tree. Such a path may be described by a sequence LRLLRL..., where an L denotes a left turn at a vertex, and an $\mathrm{R}$ denotes a right turn at a vertex. Such a path makes uniform progress in the trivalent graph. The path travels distance one in the relative space whenever the next letter in the sequence is different from the previous one, and this occurs with probability one-half. So the random walk makes progress in the relative space on average at half the rate it makes progress in the trivalent tree.

We now indicate the argument we use for more complicated mapping class groups. Imagine starting at a basepoint $x_{0}$ in hyperbolic space, and travelling some large distance $R$. The set of points you may arrive at is given by a sphere centered at $x_{0}$. Now travel another large distance $S$, as illustrated in Figure 2 .

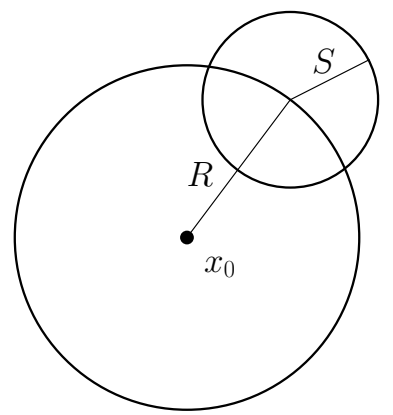

FIGURE 2. Spheres in hyperbolic space.

Due to negative curvature, nearly all the volume of the second sphere of radius $S$ lies outside the sphere of radius $R$. We wish to translate this intuition into our setting. Our space is the mapping class group $G$ with a relative metric, which we shall denote $\widehat{G}$, which is quasi-isometric to the complex of curves. Masur and Minsky [27] showed that this space is a (non-proper) $\delta$-hyperbolic space, and Klarreich 21 identified the Gromov boundary of the space as the space of foliations in $\mathcal{P} \mathcal{M F}$ which contain no closed trajectories. Let $\mu$ be a probability distribution on $G$, whose support generates a non-elementary subgroup. Kaimanovich and Masur showed that for the random walk determined by $\mu$, almost all sample paths converge to uniquely ergodic, and hence minimal, foliations and this defines a harmonic measure $\nu$ on the boundary, where $\nu(X)$ is the probability that a sample path converges to a foliation contained in the set $X$. The harmonic measure $\nu$ governs the long-time behaviour of the sample paths and is the weak- $\star$ limit of the $n$-fold convolutions of $\mu$ on $\widehat{G}$ union its boundary. In particular, if we start at the identity and consider all sample paths of length $R$, for $R$ large, the distribution of endpoints looks similar to $\nu$, at least when viewed from the identity. If we now continue the random walk for another $S$ steps, negative curvature leads us to expect that most of the new sample points will lie a definite distance further away from the origin.

In order to make this intuition precise, we need some way of comparing the harmonic measure $\nu$ with the convolution measures $\mu_{n}$. We will compare the measures on sets which are halfspaces. A halfspace $H(x, y)$ consists of all points closer (in $\widehat{G}$ ) to $y$ than $x$, and we will be most interested in halfspaces $H(1, x)$, 
where 1 is the identity element in $G$. In Section 5 we will show that the harmonic measure of a halfspace $H(1, x)$ decays exponentially in $\widehat{d}(1, x)$; i.e., there is a constant $L<1$ such that $\nu(\overline{H(1, x)}) \leqslant L^{\widehat{d}(1, x)}$, for all $x$ sufficiently far from 1, where $\overline{H(1, x)}$ is the closure of $H(1, x)$. Furthermore, we will show there is a constant $Q$ such that $\mu_{n}(H(1, x)) \leqslant Q L^{\widehat{d}(1, x)}$, for all $x$ sufficiently far from 1 , and these estimates will allow us to relate the harmonic and convolution measures. We briefly indicate why one expects these estimates to hold. First observe that there is some number $K$ such that the harmonic measure of all halfspaces $H(1, x)$, with $\widehat{d}(1, x) \geqslant K$ is bounded away from 1 , at most $1-\epsilon$, say. Then given a halfspace $H\left(1, x_{1}\right)$, where $\widehat{d}\left(1, x_{1}\right)$ is large, one may construct a nested sequence of halfspaces $H\left(1, x_{n}\right) \supset H\left(1, x_{n-1}\right) \supset \ldots \supset H\left(1, x_{1}\right)$. The number of such halfspaces is linear in $\widehat{d}\left(1, x_{i}\right)$, and furthermore, we may assume that the distance between any point in $H\left(1, x_{i}\right)$ and any point in the complement of $H\left(1, x_{i+1}\right)$ is larger than $K$. The conditional probability that a sample path converges into $H\left(1, x_{i}\right)$, given that it hits a point in the complement of $H\left(1, x_{i+1}\right)$ is at most $1-\epsilon$, so the harmonic measure of the innermost halfspace is at most $(1-\epsilon)^{n}$. In order to bound $\mu_{n}$ in terms of $\nu$, suppose a large amount of the mass of $\mu_{n}$ is contained in $H\left(1, x_{i}\right)$. Then at most $(1-\epsilon) \mu_{n}\left(H\left(1, x_{i}\right)\right)$ of the mass can escape back out into the complement of $H\left(1, x_{i+1}\right)$, so this gives the upper bound for $\mu_{n}$.

We will use the estimates described above to find a positive lower bound on the expected extra distance from 1 obtained by taking an extra $m$ steps, after a random walk of length $n$, for $m$ sufficiently large; i.e., we will show

$$
\mathbb{E}\left(\widehat{d}\left(1, w_{n+m}\right)-\widehat{d}\left(1, w_{n}\right)\right) \geqslant \delta>0 .
$$

Therefore $\mathbb{E}\left(\widehat{d}\left(1, w_{k m}\right)\right) \geqslant k \delta$, so the expected distance from the identity grows linearly, and a standard application of Kingman's subadditive ergodic theorem [20] shows that this will then be true for almost all sample paths as well.

We remark that there are distance non-increasing maps to the relative space from other useful spaces on which the mapping class group acts, such as Teichmüller space and the pants complex, so linear progress in the complex of curves implies linear progress in these spaces too. In particular, work of Duchin [6], shows that linear progress in Teichmüller space implies that for almost all sample paths there is a geodesic which the random walk tracks sublinearly, at least for the parts of the geodesic in the thick part of Teichmüller space.

In Section 2, we recall some standard definitions and set up some notation. In Section 3 we prove some useful results about halfspaces in non-proper $\delta$-hyperbolic metric spaces. As we do not assume that $\mu$ is symmetric, it will be convenient for us to know that a semi-group in the mapping class group contains a pair of independent pseudo-Anosov elements if and only if it generates a non-elementary subgroup, and we show this in Section 4, in a straightforward extension of some results of Ivanov [12. In Section 5 we show that the harmonic measure of halfspaces in the relative space decays exponentially in the distance of the halfspace from the basepoint, and we obtain the estimate for the convolution measures. Finally in Section 6 we find a positive lower bound for the expected difference between $\widehat{d}\left(1, w_{n+m}\right)$ and $\widehat{d}\left(1, w_{n}\right)$, and then apply Kingman's subadditive ergodic theorem to show that a random walk makes linear progress in the relative space. 


\section{Preliminaries}

Let $\Sigma$ be an orientable surface of finite type, i.e. a surface of genus $g$ with $p$ marked points, usually referred to as punctures. The mapping class group $G$ of $\Sigma$ consists of orientation-preserving diffeomorphisms of $\Sigma$ which preserve the punctures, modulo those isotopic to the identity. For the purposes of this paper we shall assume that $\Sigma$ is not a sphere with three or fewer punctures.

The collection of essential simple closed curves in the surface may be made into a simplicial complex, called the complex of curves, which we shall denote $\mathcal{C}(\Sigma)$. The vertices of this complex are isotopy classes of simple closed curves in $\Sigma$, and a collection of vertices spans a simplex if representatives of the curves can be realised disjointly in the surface. The complex of curves is a finite dimensional simplicial complex, but it is not locally finite. We will write $\mathcal{C}_{0}(\Sigma)$ to denote the vertices of the simplicial complex $\mathcal{C}(\Sigma)$, which is the set of isotopy classes of simple closed curves. We will write $d_{\mathcal{C}}(x, y)$ for the distance in the one-skeleton between two vertices $x$ and $y$ of the complex of curves. We will always consider the complex of curves to have a basepoint $x_{0}$, which we can take to be one of the curves corresponding to a standard generating set for the mapping class group. The mapping class group acts by simplicial isometries on the complex of curves. For certain sporadic surfaces the definition above produces a collection of disconnected points, and so a slightly different definition is used. If the surface is a torus with at most one puncture, then two vertices are connected by an edge if the corresponding simple closed curves may be isotoped to intersect transversely exactly once. If the surface is a four-punctured sphere, then two vertices are connected by an edge if the corresponding simple closed curves may be isotoped to intersect transversely in two points. In both of these cases, the resulting curve complex is isomorphic to the Farey graph.

A geodesic metric space is $\delta$-hyperbolic if every geodesic triangle is $\delta$-slim, i.e. if each edge is contained in a $\delta$-neighbourhood of the other two. Masur and Minsky 27. have shown that the complex of curves is $\delta$-hyperbolic.

The mapping class group is finitely generated, so any choice of generating set $A$ gives rise to a word metric on $G$, and any two different choices of finite generating set give quasi-isometric word metrics. Given a group $G$, and a collection of subgroups $\mathcal{H}=\left\{H_{i}\right\}_{i \in I}$, we define the relative length of a group element $g$ to be the length of the shortest word in the typically infinite generating set $A \cup \mathcal{H}$. This defines a metric on $G$ called the relative metric, which depends on the choice of subgroups $\mathcal{H}$. We will write $\widehat{G}$ to denote the group $G$ with the relative metric, which we shall also refer to as the relative space. We say a finitely generated group $G$ is weakly relatively hyperbolic, relative to a finite list of subgroups $\mathcal{H}$, if the relative space $\widehat{G}$ is $\delta$-hyperbolic.

We may consider the relative metric on the mapping class group with respect to the following collection of subgroups. Let $\left\{\alpha_{1}, \ldots, \alpha_{n}\right\}$ be a list of representatives of orbits of simple closed curves in $\Sigma$, under the action of the mapping class group. Let $H_{i}=\operatorname{fix}\left(\alpha_{i}\right)$ be the subgroup of $G$ fixing $\alpha_{i}$. Masur and Minsky [27] have shown that the resulting relative space is quasi-isometric to the complex of curves. As the complex of curves is $\delta$-hyperbolic, this shows that the mapping class group is weakly relatively hyperbolic. Klarreich 21, see also Hamenstädt [9], showed that the Gromov boundary of the complex of curves is the space $\mathcal{F}_{\text {min }}$, which we now describe. The space $\mathcal{F}_{\text {min }}$ consists of the subset of $\mathcal{P} \mathcal{M F}$ consisting of foliations 
which contain no closed trajectories, up to equivalence, where two measured foliations are equivalent if they are topologically equivalent. The topology on $\mathcal{F}_{\text {min }}$ is the induced topology from $\mathcal{P} \mathcal{M} \mathcal{F}$.

We now review some background on random walks on groups; see for example Woess 35]. Let $G$ be the mapping class group of an orientable surface of finite type, which is not a sphere with three or fewer punctures, and let $\mu$ be a probability distribution on $G$. We may use the probability distribution $\mu$ to generate a Markov chain, or random walk on $G$, with transition probabilities $p(x, y)=\mu\left(x^{-1} y\right)$. We shall always assume that we start at time zero at the identity element of the group. The path space for the random walk is the probability space $\left(G^{\mathbb{Z}_{+}}, \mathbb{P}\right)$, where $G^{\mathbb{Z}_{+}}$is the set of all infinite sequences of elements of $G$. We will write $w_{n}$ for the random variable corresponding to projection onto the $n$-th factor, which gives the position of the sample path at time $n$. The position of the random walk at time $n$ may be described as the product $m_{1} m_{2} \ldots m_{n}$, where the $m_{i}$ are the increments of the random walk; i.e., the $m_{i}$ are a sequence of independent $\mu$-distributed random variables. Therefore the distribution of random walks at time $n$ is given by the $n$-fold convolution of $\mu$, which we shall write as $\mu_{n}$, and we shall write $p^{(n)}(x, y)$ for the probability that you go from $x$ to $y$ in $n$ steps. The probability measure $\mathbb{P}$ is determined by $\mu_{n}$ using the Kolmogorov extension theorem. The reflected random walk is the walk generated by the reflected measure $\widetilde{\mu}$, where $\widetilde{\mu}(g)=\mu\left(g^{-1}\right)$. The Bernoulli shift in the space of increments of the random walk determines a measurepreserving ergodic transformation on $\left(G^{\mathbb{Z}_{+}}, \mathbb{P}\right)$ determined by $(U w)_{n}=w_{1}^{-1} w_{n+1}$.

We shall always require that the group generated by the support of $\mu$ is nonelementary, which means that it contains a pair of pseudo-Anosov elements with distinct fixed points in $\mathcal{P} \mathcal{M F}$, the space of projective measured foliations on the surface. We do not assume that the probability distribution $\mu$ is symmetric, so the group generated by the support of $\mu$ may be strictly larger than the semi-group generated by the support of $\mu$. Finally, we shall always assume that the probability distribution $\mu$ has finite first moment with respect to the word metric on $G$.

In 25, we showed that it followed from results of Kaimanovich and Masur [14] and Klarreich [21] that a sample path converges almost surely to a uniquely ergodic foliation in the Gromov boundary of the relative space. This gives a measure $\nu$ on $\mathcal{F}_{\text {min }}$, known as harmonic measure. The harmonic measure $\nu$ is $\mu$-stationary, i.e.

$$
\nu(X)=\sum_{g \in G} \mu(g) \nu\left(g^{-1} X\right) .
$$

Theorem 2.1 (14,21,25]). Consider a random walk on the mapping class group of an orientable surface of finite type, which is not a sphere with three or fewer punctures, determined by a probability distribution $\mu$ such that the group generated by the support of $\mu$ is non-elementary. Then a sample path $\left\{w_{n}(\omega)\right\}$ converges to a uniquely ergodic foliation in the Gromov boundary $\mathcal{F}_{\text {min }}$ of the relative space $\widehat{G}$ almost surely, and the distribution of limit points on the boundary is given by a unique $\mu$-stationary, non-atomic measure $\nu$ on $\mathcal{F}_{\text {min }}$.

We remark that the measure $\nu$ is supported on the uniquely ergodic foliations, which are a subset of $\mathcal{P} \mathcal{M F}$, so we may think of $\nu$ as a measure on $\mathcal{P} \mathcal{M} \mathcal{F}$, with zero weight on all the non-uniquely ergodic measures. 


\section{HALFspaCes}

In this section we give detailed proofs of various useful properties of halfspaces in a non-locally compact $\delta$-hyperbolic space. These properties are presumably well known for locally compact $\delta$-hyperbolic spaces, but we provide complete proofs to verify that these properties hold in the non-locally compact case. For consistency with the other sections of this paper, we will denote our non-locally compact $\delta$ hyperbolic metric space by $\widehat{G}$, and we will write $\widehat{d}(x, y)$ for the distance in $\widehat{G}$ between two points $x$ and $y$. Furthermore, we will always have a distinguished base point, which we shall call 1 .

Two points $a$ and $b$ in a metric space define a halfspace $H(a, b)$ consisting of all those points which are at least as close to $b$ as to $a$, i.e. $H(a, b)=\{x \in \widehat{G} \mid$ $\widehat{d}(x, b) \leqslant \widehat{d}(x, a)\}$. The two main results of this section are Propositions 3.9 and 3.10, which we now briefly describe. Proposition 3.9 says if two halfspaces $H(1, x)$ and $H(1, y)$ are small and far apart, then any other small halfspace hits at most one of $H(1, x)$ or $H(1, y)$. The halfspaces $H(1, x)$ and $H(1, y)$ are small if $\widehat{d}(1, x)$ and $\widehat{d}(1, y)$ are large, and they are far apart if the geodesic $[x, y]$ passes close to the basepoint 1. In this case any other halfspace $H(1, z)$ hits at most one of $H(1, x)$ or $H(1, y)$, as long as $\widehat{d}(1, z)$ is sufficiently large. Proposition 3.10 says that given a halfspace $H(1, x)$, we may choose a point $y$ on a geodesic $[1, x]$ close to $x$ such that the halfspace $H(1, x)$ is contained in the halfspace $H(1, y)$. We also obtain specific bounds on how large the halfspace $H(1, x)$ appears when viewed from any point in $H(y, 1)$, and similar bounds on how large the halfspace $H(y, 1)$ appears when viewed from any point in $H(1, x)$. To be more precise, we show that for any point $a$ in $H(y, 1)$, the halfspace $H(1, x)$ is contained in a halfspace $H(a, b)$, with an explicit lower bound on $\widehat{d}(a, b)$. Furthermore, for any point $b$ in $H(1, x)$, there is a halfspace $H(b, a)$ such that $H(y, 1) \subset H(b, a)$, again, with an explicit lower bound on $\widehat{d}(a, b)$.

We begin with some elementary observations about nearest point projections. In a $\delta$-hyperbolic space, nearest point projections onto quasi-convex sets are coarsely well defined. We now show that for any set $X$, the $K$-neighbourhoods of $X$, and a particular choice of shortest path to $X$, have bounded intersection.

Proposition 3.1. Let $X$ be a set, and let $[z, p]$ be a minimal length geodesic from a point $z$ to $X$. Then the intersection of the $K$-neighbourhoods of $X$ and $[z, p]$ is contained in a $3 K$-neighbourhood of $p$.

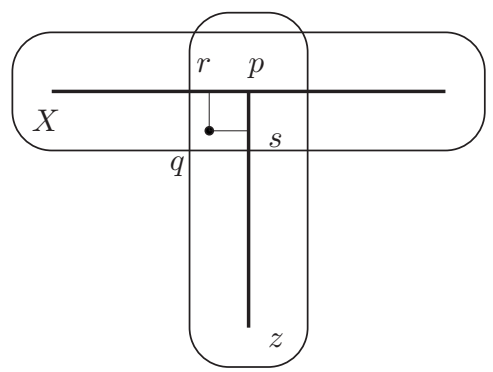

Figure 3. A shortest path from $z$ to $X$. 
Proof. Choose a point $q$ in the intersection of $N_{K}(X)$ and $N_{K}([z, p])$. There are points $r \in X$ and $s \in[z, p]$ such that $\widehat{d}(r, q) \leqslant K$ and $\widehat{d}(s, q) \leqslant K$, as illustrated in Figure 3. If $\widehat{d}(p, q)>3 K$, then as $\widehat{d}(s, q) \leqslant K$, this implies that $\widehat{d}(p, s)>$ $2 K$. Therefore $r$ is a closer point on $[x, y]$ to $s$, and hence to $z$, than $p$, which contradicts the fact that $p$ is a closest point on $X$ to $z$. So $\widehat{d}(p, q) \leqslant 3 K$, and so $N_{K}(X) \cap N_{K}([z, p]) \subset N_{3 K}(p)$, as required.

This shows that, up to additive error, the shortest way to get from a point $z$ to a point $x$ on a geodesic is to head to the closest point to $z$ on the geodesic, and then run along the geodesic to $x$. We will make extensive use of this fact, so we record it here as a proposition.

Proposition 3.2. Let $[x, y]$ be a geodesic from $x$ to $y$, and let $p$ be a closest point on $[x, y]$ to $z$. Any geodesic $[x, z]$ from $x$ to $z$ intersects a $3 \delta$-neighbourhood of $p$, so $[x, p] \cup[p, z]$ is contained in a $3 \delta$-neighbourhood of $[x, z]$. In particular $\widehat{d}(x, p)+$ $\widehat{d}(p, z)-6 \delta \leqslant \widehat{d}(x, z) \leqslant \widehat{d}(x, p)+\widehat{d}(p, z)$.

Proof. The right hand inequality is just the triangle inequality. We now justify the left hand inequality. By thin triangles, any geodesic $[x, z]$ from $x$ to $z$ is contained in a $\delta$-neighbourhood of the union of geodesics $[x, p]$ and $[p, z]$. If $[p, x]$ and $[p, z]$ had long initial segments that fellow travelled, then the distance from $x$ to $z$ might be much shorter than the sum of the distances from $x$ to $p$ and from $z$ to $p$. However, this would contradict the fact that $p$ was a closest point on $[x, y]$ to $z$. To be precise, Proposition 3.1 implies that the intersection of the $\delta$-neighbourhoods of $[x, p]$ and $[p, z]$ is contained in a $3 \delta$-neighbourhood of $p$. This means that any geodesic from $z$ to $x$ must pass within $3 \delta$ of $p$, so $\widehat{d}(z, p)+\widehat{d}(p, x)-6 \delta \leqslant \widehat{d}(z, x)$, as required.

An immediate consequence of Proposition 3.2 is the following quantitative version of the fact that the nearest point projection is coarsely well defined.

Proposition 3.3. Let $p$ and $q$ be nearest points to $z$ on a geodesic $[x, y]$. Then $\widehat{d}(p, q) \leqslant 6 \delta$.

Let $a$ and $b$ have nearest point projections $p$ and $q$ onto a geodesic $[x, y]$. We now show that if $\widehat{d}(p, q)>14 \delta$ apart, then, up to additive error, the geodesic from $a$ to $b$ goes from $a$ to $p$, then runs along the geodesic from $p$ to $q$, and then heads back out to $b$.

Proposition 3.4. Let $[x, y]$ be a geodesic and let $p$ be a closest point on $[x, y]$ to $a$, and let $q$ be a closest point on $[x, y]$ to $b$. If $\widehat{d}(p, q)>14 \delta$, then $\widehat{d}(a, b) \geqslant$ $\widehat{d}(a, p)+\widehat{d}(p, q)+\widehat{d}(q, b)-24 \delta$.

Proof. Let $[x, y]$ be a geodesic from $x$ to $y$. Let $[a, p]$ be a minimal length geodesic from $a$ to $[x, y]$, and let $[b, q]$ be a minimal length geodesic from $b$ to $[x, y]$. First we show that $N_{2 \delta}([a, p])$ and $N_{2 \delta}([b, q])$ are disjoint. Suppose not. Then let $r$ be a point in $N_{2 \delta}([a, p]) \cap N_{2 \delta}([b, q])$. Then there are points $s \in[a, p]$ and $t \in[b, q]$ such that $\widehat{d}(s, r) \leqslant 2 \delta$ and $\widehat{d}(t, r) \leqslant 2 \delta$, as illustrated in Figure 4 . 


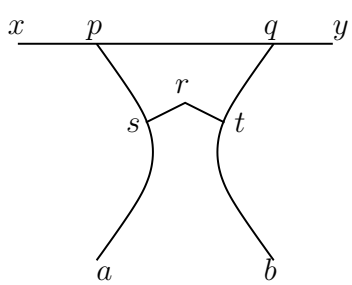

Figure 4. Shortest paths from $a$ and $b$ to $[x, y]$. that

By the triangle inequality, $\widehat{d}(t, p) \leqslant \widehat{d}(t, s)+\widehat{d}(s, p)$. As $\widehat{d}(t, s) \leqslant 4 \delta$, this shows

$$
\widehat{d}(t, p) \leqslant 4 \delta+\widehat{d}(s, p) .
$$

By Proposition 3.2. the path from $t$ to $p$ via $q$ is almost a geodesic, i.e.

$$
\widehat{d}(t, q)+\widehat{d}(q, p)-6 \delta \leqslant \widehat{d}(t, p) .
$$

Combining (3.1) and (3.2), we obtain

$$
\widehat{d}(t, q)+\widehat{d}(q, p)-6 \delta \leqslant \widehat{d}(s, p)+4 \delta .
$$

As $[a, p]$ is a minimal length geodesic from $a$ to $[x, y]$, it is also a minimal length geodesic from any point on $[a, p]$ to $[x, y]$. Therefore $p$ is a closest point on $[x, y]$ to $s$, so the distance from $s$ to $p$ is less than or equal to the distance from $s$ to $[x, y]$ by a path through $t$. This implies that

$$
\widehat{d}(s, p) \leqslant 4 \delta+\widehat{d}(t, q) .
$$

Combining (3.3) and (3.4), and subtracting $\widehat{d}(t, q)$ from both sides, implies that $\widehat{d}(p, q) \leqslant 14 \delta$. However we assumed that $\widehat{d}(p, q)>14 \delta$, so this implies that $N_{2 \delta}([a, p])$ and $N_{2 \delta}([b, q])$ are in fact disjoint.

By thin triangles, any geodesic $[a, b]$ is contained in $2 \delta$-neighbourhood of $[a, p] \cup$ $[p, q] \cup[q, b]$. By Proposition [3.1, the intersection of $N_{2 \delta}([a, p])$ with $N_{2 \delta}([x, y])$ is contained in a $6 \delta$-neighbourhood of $p$. Similarly, the intersection of $N_{2 \delta}([b, q])$ with $N_{2 \delta}([x, y])$ is contained in a $6 \delta$-neighbourhood of $q$. As the remaining parts of the $2 \delta$-neighbourhoods outside $N_{6 \delta}(p)$ and $N_{6 \delta}(q)$ are disjoint, this means that $\widehat{d}(a, b) \geqslant \widehat{d}(a, p)+\widehat{d}(p, q)+\widehat{d}(q, b)-24 \delta$, as required.

We now show that the nearest point projection to a connected subgeodesic of a geodesic is coarsely equivalent to the nearest point projection to the original geodesic, followed by the nearest point projection to the subgeodesic.

Proposition 3.5. Let $\rho_{1}$ be the nearest point projection onto a geodesic $[a, b]$, and let $\rho_{2}$ be the nearest point projection onto a subgeodesic $[c, d] \subset[a, b]$. Then there is a constant $K_{1}$, which only depends on $\delta$, such that $\widehat{d}\left(\rho_{2}(x), \rho_{2}\left(\rho_{1}(x)\right)\right) \leqslant K_{1}$, for any point $x$.

Proof. Let $p$ be the nearest point projection of $x$ to $[a, b]$, i.e. $p=\rho_{1}(x)$. If $q$ is another point on $[a, b]$, then the path from $x$ to $q$ via $p$ is almost a geodesic, Proposition 3.2 so $\widehat{d}(x, p)+\widehat{d}(p, q)-6 \delta \leqslant \widehat{d}(x, q) \leqslant \widehat{d}(x, p)+\widehat{d}(p, q)$. Therefore, if $p$ lies in the subgeodesic $[c, d]$, then $\pi_{2}(p)=p$, and any point in $[c, d]$ further 
than $6 \delta$ from $p$ is further away from $x$ than $p$. On the other hand, if $p$ lies outside $[c, d]$, then $\rho_{2}(p)$ is equal to one of the endpoints, which we may assume is $c$, up to relabelling. Again, Proposition 3.4 implies that any point on $[c, d]$ more than $6 \delta$ away from $c$ is further away from $x$ than $c$. So we may choose $K_{1}$ to be $7 \delta$.

We now show that if a path $\gamma$ lies in a bounded neighbourhood of a geodesic, then the nearest point projections of any point to the path and to the geodesic are a bounded distance apart.

Proposition 3.6. Let $[x, y]$ be a geodesic, and let $\gamma$ be any path from $x$ to $y$ contained in a K-neighbourhood of $[x, y]$. For any point $z$, let $p$ be a closest point on $[x, y]$ to $z$, and let $q$ be a closest point on $\gamma$ to $z$. Then $\widehat{d}(p, q) \leqslant 3 K+6 \delta$.

Proof. Let $p$ be the closest point to $z$ on $[x, y]$. As $\gamma$ is a path from $[x, y]$ contained in a $K$-neighbourhood of $[x, y]$, there is a point $p^{\prime}$ in $\gamma$ such that $\widehat{d}\left(p, p^{\prime}\right) \leqslant K$, and hence $\widehat{d}\left(p^{\prime}, z\right) \leqslant \widehat{d}(z, p)+K$. Let $q$ be the closest point on $\gamma$ to $z$, and let $q^{\prime}$ be the closest point on $[x, y]$ to $q$, so the distance from $\widehat{d}\left(q, q^{\prime}\right) \leqslant K$. This is illustrated in Figure 5 .

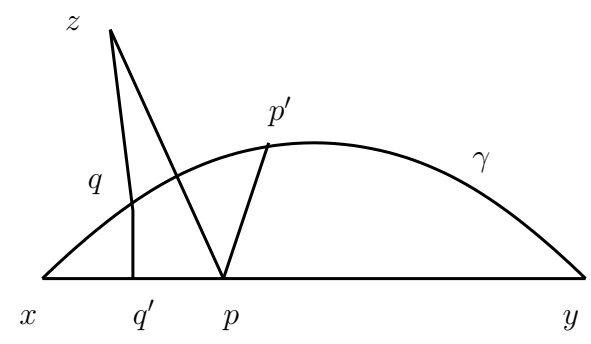

Figure 5. A path close to a geodesic $[x, y]$.

By Proposition 3.2, the path from $z$ to $q^{\prime}$ via $p$ is almost a geodesic, so $\widehat{d}\left(z, q^{\prime}\right) \geqslant$ $\widehat{d}(z, p)+\widehat{d}\left(p, q^{\prime}\right)-6 \delta$, which implies that $\widehat{d}(z, q) \geqslant \widehat{d}(z, p)+\widehat{d}\left(p, q^{\prime}\right)-6 \delta-K$. Therefore if $\widehat{d}\left(p, q^{\prime}\right)>2 K+6 \delta$, then $\widehat{d}(z, q) \geqslant \widehat{d}\left(z, p^{\prime}\right)$, which contradicts our choice of $q$ as a closest point on $\gamma$ to $z$. Therefore $\widehat{d}(p, q) \leqslant 3 K+6 \delta$, as required.

Recall that two points $x$ and $y$ define a halfspace $H(x, y)$. We now show that the image of the halfspace under the nearest point projection to a geodesic $[x, y]$ between $x$ and $y$ is contained in a bounded neighbourhood of the half-segment of $[x, y]$ closest to $y$. As a partial converse, we show that if the nearest point projection of $z$ lies sufficiently close to $y$, then $z \in H(x, y)$.

Proposition 3.7. Let $z \in H(x, y)$, and let $p$ be the nearest point to $z$ on a geodesic $[x, y]$. Then $\widehat{d}(y, p) \leqslant \frac{1}{2} \widehat{d}(x, y)+3 \delta$. Conversely, if $\widehat{d}(y, p) \leqslant \frac{1}{2} \widehat{d}(x, y)-3 \delta$, then $z \in H(x, y)$.

Proof. Let $z$ be a point in the halfspace $H(x, y)$, let $[x, y]$ be a geodesic from $x$ to $y$, and let $p$ be a closest point on $[x, y]$ to $z$. This is illustrated in Figure 6 


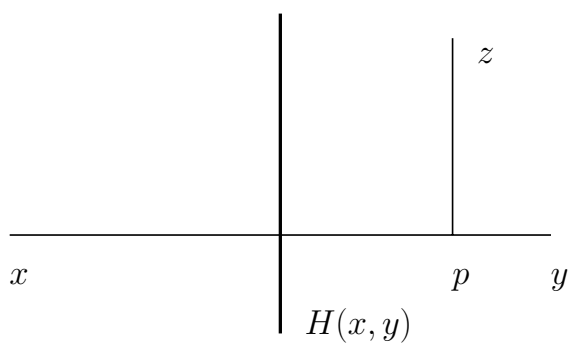

FiguRE 6. A point $z$ in the halfspace $H(x, y)$.

The point $z$ lies in the halfspace $H(x, y)$, so $\widehat{d}(z, y) \leqslant \widehat{d}(z, x)$. By the triangle inequality, the distance from $z$ to $x$ is less than or equal to the distance from $z$ to $x$ via $p$, which implies

$$
\widehat{d}(z, y) \leqslant \widehat{d}(z, p)+\widehat{d}(p, x) .
$$

By Proposition 3.2, the path from $z$ to $y$ via $p$ is close to being a geodesic, i.e. $\widehat{d}(z, p)+\widehat{d}(p, y)-6 \delta \leqslant \widehat{d}(z, y)$. Therefore

$$
\widehat{d}(z, p)+\widehat{d}(p, y)-6 \delta \leqslant \widehat{d}(z, p)+\widehat{d}(p, x) .
$$

We may subtract $\widehat{d}(z, p)$ from both sides. As $p$ lies on the geodesic $[x, y]$, the distance from $x$ to $y$ is equal to the distance from $x$ to $p$ plus the distance from $p$ to $y$, i.e. $\widehat{d}(x, y)=\widehat{d}(x, p)+\widehat{d}(p, y)$. This gives the required inequality, $\widehat{d}(p, y) \leqslant \frac{1}{2} \widehat{d}(x, y)+3 \delta$.

For the converse, note that if $z$ does not lie in the halfspace $H(x, y)$, then $z$ lies in the halfspace $H(y, x)$, so $\widehat{d}(p, x) \leqslant \frac{1}{2} \widehat{d}(x, y)+3 \delta$. The point $p$ lies on the geodesic $[x, y]$, so $\widehat{d}(p, x)=\widehat{d}(x, y)-\widehat{d}(p, y)$, which implies $\frac{1}{2} \widehat{d}(x, y) \leqslant \widehat{d}(p, y)+3 \delta$. Therefore, if $\widehat{d}(y, p) \leqslant \frac{1}{2} \widehat{d}(x, y)-3 \delta$, then this implies $z \in H(x, y)$, as required.

We have shown that the image of the nearest point projection of a halfspace $H(x, y)$ to $[x, y]$ is close to being half of this geodesic segment. If a geodesic segment $[x, z]$ fellow travels with $[x, y]$ for a sufficiently large initial segment, then we can estimate the image of the nearest point projection of $H(x, y)$ onto $[x, z]$. This will be the case as long as the nearest point projection of $z$ to $[x, y]$ is sufficiently far from $x$. We now make this precise in the following proposition.

Proposition 3.8. There are constants $K_{2}$ and $K_{3}$, which only depend on $\delta$, such that if $z$ has nearest point projection $p$ to a geodesic $[x, y]$, and $\widehat{d}(p, x) \geqslant \frac{1}{2} \widehat{d}(x, y)+$ $K_{2}$, then the nearest point projection of $H(y, x)$ to $[x, z]$ is at a distance at most $\frac{1}{2} \widehat{d}(x, y)+K_{3}$ from $x$.

Proof. We shall choose $K_{2}$ to be $27 \delta$ and $K_{3}$ to be $18 \delta$. Let $H(y, x)$ be the halfspace defined by the pair of points $x$ and $y$, and let $[x, y]$ be a geodesic from $x$ to $y$. Let $p$ be a nearest point to $z$ on $[x, y]$. Let $a \in H(y, x)$, and let $q$ be the closest point on $[x, y]$ to $a$. This is illustrated in Figure 7 


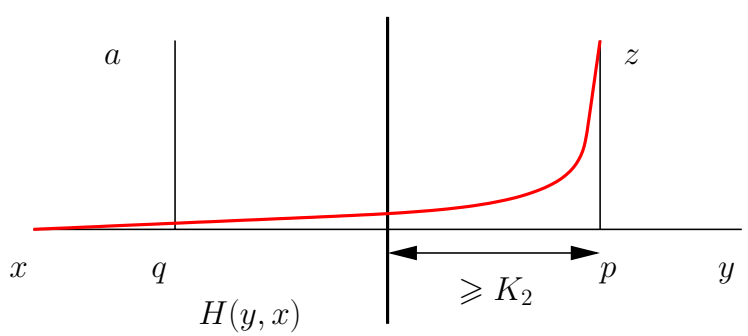

Figure 7. Closest point projection of $H(y, x)$ onto $[x, z]$.

By Proposition 3.7 the nearest point projection maps the halfspace $H(y, x)$ to roughly half the geodesic $[x, y]$. This implies that $\widehat{d}(q, x) \leqslant \frac{1}{2} \widehat{d}(x, y)+3 \delta$, so $\widehat{d}(q, p) \geqslant K_{2}-3 \delta$. Let $t$ be a point on $[p, z]$. Then as $K_{2} \geqslant 17 \delta$, Proposition 3.4 implies that $\widehat{d}(a, t) \geqslant \widehat{d}(a, q)+\widehat{d}(q, p)+\widehat{d}(p, t)-24 \delta$. As we chose $K_{2}$ to be $27 \delta$, this implies $\widehat{d}(p, q) \geqslant 24 \delta$, and so the closest point to $a$ on $[x, p] \cup[p, z]$ lies on $[x, p]$. As $[x, p] \subset[x, y]$, Proposition 3.7 implies that the nearest point projection of $H(y, x)$ to $[x, p] \cup[p, z]$ is at a distance at most $\frac{1}{2} \widehat{d}(x, y)+3 \delta$ from $x$.

Let $r$ be the nearest point to $a$ on the geodesic $[x, z]$, and let $s$ be the nearest point to $a$ on the path $[x, p] \cup[p, z]$. By Proposition 3.2. the path from $x$ to $z$ via $p$ is contained in a $3 \delta$-neighbourhood of a geodesic $[x, z]$, so as nearest point projections to close paths are close, Proposition [3.6 this implies $\widehat{d}(r, s) \leqslant 3(3 \delta)+6 \delta=15 \delta$. Therefore the nearest point projection of $H(y, x)$ to $[x, z]$ is at a distance at most $\frac{1}{2} \widehat{d}(x, y)+18 \delta$ from $x$, and so we may choose $K_{3}$ to be $18 \delta$.

We now show that if $H(1, x)$ and $H(1, y)$ are two halfspaces far from the origin, such that the geodesic from $[x, y]$ passes close to the origin, then any other halfspace far from the origin hits at most one of $H(1, x)$ or $H(1, y)$.

Proposition 3.9. There are constants $K_{4}$ and $K_{5}$, which only depend on $\delta$, such that for any geodesic $[x, y]$ with $\widehat{d}(1, x)$ and $\widehat{d}(1, y)$ at least $2 \widehat{d}(1,[x, y])+K_{4}$, and for any point $z$ with $\widehat{d}(1, z) \geqslant 2 \widehat{d}(1,[x, y])+K_{5}$, the halfspace $H(1, z)$ hits at most one of the halfspaces $H(1, x)$ and $H(1, y)$.

We remark that the hypotheses may be restated in terms of the Gromov product, $(x \mid y)=\frac{1}{2}(\widehat{d}(1, x)+\widehat{d}(1, y)-\widehat{d}(x, y))$, as in a $\delta$-hyperbolic space the distance from the basepoint 1 to a geodesic $[x, y]$ is coarsely equivalent to the Gromov product of $x$ and $y$.

Proof. We shall choose $K_{4}$ to be $2 K_{2}+K_{3}+42 \delta$, and $K_{5}$ to be $24 \delta$. Let $[x, y]$ be a geodesic from $x$ to $y$, and let $p$ be a closest point on $[x, y]$ to 1 . It will be convenient to use the following form of our assumption that the distance from 1 to $x$ is larger than twice the distance from 1 to $p$ :

$$
\frac{1}{2} \widehat{d}(1, x) \geqslant \widehat{d}(1, p)+\frac{1}{2} K_{4} .
$$

Let $r$ be the nearest point on $[1, x]$ to $y$, as illustrated in Figure 8 We start by showing that $r$ is a bounded distance from $p$. By Proposition 3.2 the path from $y$ to 1 via $p$ is almost a geodesic, so if $t$ is a point on $[1, p]$, then $\widehat{d}(t, y) \geqslant$ 
$\widehat{d}(y, p)+\widehat{d}(p, t)-6 \delta$. This implies that the closest point projection of $y$ to $[x, p] \cup[p, 1]$ is at a distance at most $6 \delta$ from $p$. As the path $[1, p] \cup[p, x]$ lies in a $3 \delta$-neighbourhood of the geodesic $[1, x]$, and as the nearest point projections to close paths are close, Proposition 3.6, this implies that

$$
\widehat{d}(r, p) \leqslant 3(3 \delta)+6 \delta+6 \delta=21 \delta .
$$

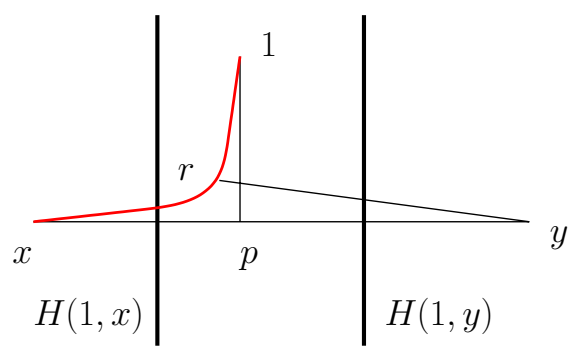

Figure 8. Disjoint halfspaces.

In order to use Proposition 3.8 to estimate the size of the nearest point projection of the halfspace $H(1, x)$ to the geodesic $[x, y]$ we need to show that $r$ is closer to 1 than $x$. As $r$ lies on a geodesic from 1 to $x$,

$$
\widehat{d}(x, r)=\widehat{d}(1, x)-\widehat{d}(1, r) .
$$

Using our initial assumption, line (3.5), we may rewrite this as

$$
\widehat{d}(x, r) \geqslant \frac{1}{2} \widehat{d}(1, x)+\widehat{d}(1, p)-\widehat{d}(1, r)+\frac{1}{2} K_{4} .
$$

Using the bound for $\widehat{d}(r, p)$ from line (3.6) and the triangle inequality, we obtain

$$
\widehat{d}(x, r) \geqslant \frac{1}{2} \widehat{d}(1, x)+\frac{1}{2} K_{4}-21 \delta .
$$

Therefore we may apply Proposition 3.8 , as we chose $K_{4} \geqslant 2 K_{2}+42 \delta$. This implies that the nearest point projection of $H(1, x)$ to $[x, y]$ is contained in a $\left(\frac{1}{2} \widehat{d}(1, x)+K_{3}\right)$ neighbourhood of $x$.

We now show that the nearest point projection of $H(1, x)$ to $[x, y]$ is a definite distance away from $p$. Let $s$ be a point in the nearest point projection of $H(1, x)$ to $[x, y]$, so we have just shown that $\widehat{d}(s, x) \leqslant \frac{1}{2} \widehat{d}(1, x)+K_{3}$. As $s$ lies on the geodesic $[x, p]$, this implies

$$
\widehat{d}(s, p) \geqslant \widehat{d}(x, p)-\frac{1}{2} \widehat{d}(1, x)-K_{3}
$$

Using the triangle inequality applied to going from 1 to $x$ via $p$, we obtain

$$
\widehat{d}(s, p) \geqslant \widehat{d}(1, x)-\widehat{d}(1, p)-\frac{1}{2} \widehat{d}(1, x)-K_{3} .
$$

Our initial assumption (3.5) now implies that $\widehat{d}(s, p) \geqslant K_{4}-K_{3}$, which is greater than zero if $K_{4}>K_{3}$. In fact $\widehat{d}(s, p) \geqslant 14 \delta$, as we chose $K_{4}$ to be $2 K_{2}+K_{3}+42 \delta$.

Our hypotheses are symmetric in $x$ and $y$, so this also implies identical results for the image of the nearest point projection of $H(1, y)$ to $[x, y]$. In particular, this implies that the nearest point projections of $H(1, x)$ and $H(1, y)$ to the geodesic $[x, y]$ are disjoint. 
We now consider a halfspace $H(1, z)$ which intersects both $H(1, x)$ and $H(1, y)$, and we show that there is an upper bound on $\widehat{d}(1, z)$. Assume that $H(1, z)$ intersects both $H(1, x)$ and $H(1, y)$. Let $q$ be a closest point on $[x, y]$ to $z$. Up to relabelling $x$ and $y$, we may assume that $q \in[p, y]$. This is illustrated in Figure 9

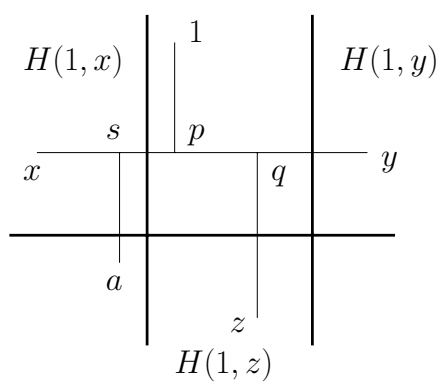

Figure 9. Three halfspaces.

Let $a$ be a point lying in the intersection of $H(1, x)$ and $H(1, z)$, so in particular

$$
\widehat{d}(a, z) \leqslant \widehat{d}(1, a) .
$$

Let $s$ be a nearest point on $[x, y]$ to $a$. We showed above that $\widehat{d}(s, p)>14 \delta$, and therefore $\widehat{d}(s, q)>14 \delta$. As $s$ and $q$ are the nearest point projections of $a$ and $z$ onto the geodesic $[x, y]$, and $\widehat{d}(s, q)>14 \delta$, Proposition 3.4 implies that the path from $a$ to $z$ via $s$ and $q$ is close to being a geodesic, i.e. $\widehat{d}(a, z) \geqslant \widehat{d}(a, s)+\widehat{d}(s, p)+$ $\widehat{d}(p, q)+\widehat{d}(q, z)-24 \delta$. Together with line (3.7), this implies

$$
\widehat{d}(a, s)+\widehat{d}(s, p)+\widehat{d}(p, q)+\widehat{d}(q, z)-24 \delta \leqslant \widehat{d}(1, a) .
$$

By the triangle inequality, $\widehat{d}(1, a) \leqslant \widehat{d}(1, p)+\widehat{d}(p, s)+\widehat{d}(s, a)$, which gives

$$
\widehat{d}(a, s)+\widehat{d}(s, p)+\widehat{d}(p, q)+\widehat{d}(q, z)-24 \delta \leqslant \widehat{d}(1, p)+\widehat{d}(p, s)+\widehat{d}(s, a) .
$$

Adding $\widehat{d}(1, p)-\widehat{d}(a, s)-\widehat{d}(s, p)$ to both sides, we obtain

$$
\widehat{d}(1, p)+\widehat{d}(p, q)+\widehat{d}(q, z) \leqslant 2 \widehat{d}(1, p)+24 \delta .
$$

Using the triangle inequality, this shows that $\widehat{d}(1, z) \leqslant 2 \widehat{d}(1, p)+24 \delta$. So we may choose $K_{5}$ to be $24 \delta$.

We now show that if $H(1, x)$ is a halfspace far from the origin, then it is contained in a halfspace $H(1, y)$, with $y$ a bounded distance closer to the origin, and we find an explicit lower bound on the distance between $H(1, x)$ and $H(y, 1)$. Furthermore, we show that for any point $a$ in $H(y, 1)$, there is a point $b$ such that the halfspace $H(a, b)$ contains $H(1, x)$, with an explicit lower bound on $\widehat{d}(a, b)$. Similarly, we show that for any point $b$ in $H(1, x)$, there is a point $a$ such that $H(y, 1) \subset H(b, a)$, again with an explicit lower bound on $\widehat{d}(a, b)$.

Proposition 3.10. There are constants $K_{6}$ and $K_{7}$, which only depend on $\delta$, such that for any positive number $A$, and any point $x$ with $\widehat{d}(1, x) \geqslant K_{6}+4 A$, there is a point $y$ on $[1, x]$ with $\widehat{d}(1, y)=\widehat{d}(1, x)-2 K_{7}-2 A$, such that $H(1, x) \subset H(1, y)$. 
Furthermore, for any point a in $H(y, 1)$, there is a point $b$ in $H(1, x)$, with $\widehat{d}(a, b) \geqslant$ $2 A$, and $H(1, x) \subset H(a, b)$. Also, for any point $b$ in $H(1, x)$, there is a point $a$ in $H(y, 1)$ with $\widehat{d}(a, b) \geqslant 2 A$, and $H(y, 1) \subset H(b, a)$.

Proof. We shall choose $K_{7}$ to be $98 \delta+2 K_{1}$ and $K_{6}$ to be $2 K_{7}+6 \delta$. We first show that $H(1, x) \subset H(1, y)$. Let $p$ be a point in the nearest point projection of $H(y, 1)$ to $[1, x]$, and let $q$ be a point in the nearest point projection of $H(1, x)$ to $[1, x]$. Applying Proposition 3.7 to the halfspace $H(1, y)$ gives

$$
\widehat{d}(1, p) \leqslant \frac{1}{2} \widehat{d}(1, y)+3 \delta .
$$

Using our assumption that $\widehat{d}(1, y)=\widehat{d}(1, x)-2 K_{7}-2 A$ implies

$$
\widehat{d}(1, p) \leqslant \frac{1}{2} \widehat{d}(1, x)+3 \delta-A-K_{7} .
$$

Now applying Proposition 3.7 to the halfspace $H(1, x)$, we obtain

$$
\widehat{d}(q, 1) \geqslant \frac{1}{2} \widehat{d}(1, x)-3 \delta .
$$

Comparing (3.8) and (3.9) shows that if $K_{7} \geqslant 13 \delta$, then $\widehat{d}(p, q) \geqslant 7 \delta$, and so $H(1, x)$ and $H(y, 1)$ are disjoint, which implies $H(1, x) \subset H(1, y)$, as required.

We now prove the second statement in Proposition 3.10 .

Claim 3.11. For any point $a$ in $H(y, 1)$ there is a point $b$ in $H(1, x)$, with $\widehat{d}(a, b) \geqslant$ $2 A$, and $H(1, x) \subset H(a, b)$.

Proof. Given a point $a$ in $H(y, 1)$, we will choose a point $b$ in the geodesic $[1, x]$, sufficiently far from $a$, and then show that $H(1, x) \subset H(a, b)$ by showing that the nearest point projections of $H(1, x)$ and $H(b, a)$ to $[1, x]$ are sufficiently far apart.

Let $a$ be a point in the halfspace $H(y, 1)$, let $p$ be a nearest point projection of $a$ to $[1, x]$ and let $b$ be a point on the geodesic $[1, x]$, a distance $\widehat{d}(1, p)+K_{7}$ from $x$. This is illustrated in Figure 10.

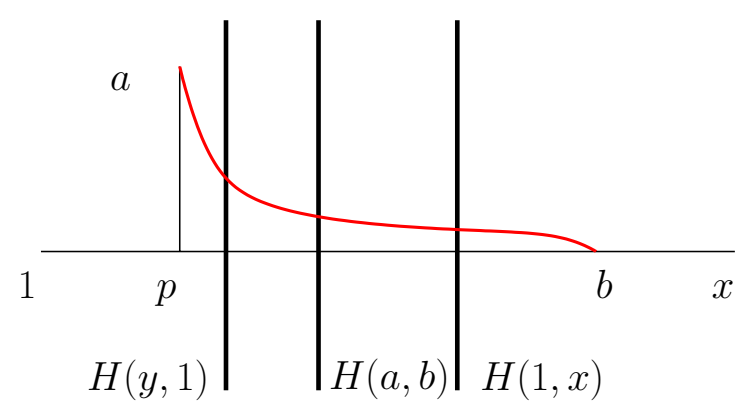

FIgURE 10. The halfspace $H(1, x)$ is contained in the halfspace $H(a, b)$.

We first find a lower bound for the distance between $a$ and $b$. Using Proposition 3.2. which says that the path $[a, p] \cup[p, b]$ is contained in a $3 \delta$-neighbourhood of $[a, b]$, and the triangle inequality, we obtain

$$
\widehat{d}(a, b) \geqslant \widehat{d}(p, b)-6 \delta .
$$

Let $z$ be the midpoint of $[1, x]$. Then $\widehat{d}(p, z) \geqslant K_{7}+A-3 \delta$, by Proposition 3.7. and our assumption on the distance between the halfspaces $H(1, x)$ and $H(1, y)$. 
Our choice of $b$ then implies $\widehat{d}(z, b) \geqslant A-3 \delta$. Therefore $\widehat{d}(p, b) \geqslant K_{7}+2 A-12 \delta$, and this is at least $2 A$, as required, as we chose $K_{7} \geqslant 12 \delta$.

We now show that the halfspace $H(1, x)$ is contained in the halfspace $H(a, b)$. As the nearest point projection is coarsely well defined, Proposition 3.3. it suffices to show that the nearest point projections of $H(1, x)$ and $H(b, a)$ to $[1, x]$ are distance at least $7 \delta$ apart. By Proposition 3.7, if $r$ is a point in the nearest point projection of $H(1, x)$ to $[1, x]$, then

$$
\widehat{d}(r, x) \leqslant \frac{1}{2} \widehat{d}(1, x)+3 \delta .
$$

Similarly, the nearest point projection of $H(b, a)$ to $[a, b]$ is at a distance at least $\frac{1}{2} \widehat{d}(a, b)-3 \delta$ from $b$. Let $s$ be a closest point on $[a, b]$ to $p$. By Proposition 3.5, if $r$ is a point in the nearest point projection of $H(b, a)$ to the subgeodesic $[s, b]$ of $[a, b]$, then

$$
\widehat{d}(r, b) \geqslant \frac{1}{2} \widehat{d}(a, b)-3 \delta-K_{1} .
$$

By Proposition 3.2, $\widehat{d}(s, p) \leqslant 3 \delta$, and the path $[s, p] \cup[p, b]$ is contained in a $3 \delta$ neighbourhood of $[s, b]$. Therefore, as the nearest point projections to close paths are close, Proposition [3.6, this implies that if $r$ is a nearest point in $[s, p] \cup[p, b]$ to $H(b, a)$, then

$$
\widehat{d}(r, b) \geqslant \frac{1}{2} \widehat{d}(a, b)-18 \delta-K_{1} .
$$

The nearest point projection onto the path $[s, p] \cup[p, b]$ is the same as the nearest point projection onto the path $[p, s] \cup[s, p] \cup[p, b]$, and this latter path is contained in a $3 \delta$-neighbourhood of $[p, b]$, so again applying Proposition [3.6. if $r$ lies in the nearest point projection of $H(b, a)$ to $[p, b]$, then

$$
\widehat{d}(r, b) \geqslant \frac{1}{2} \widehat{d}(a, b)-33 \delta-K_{1} .
$$

Using (3.10), and the fact that $[p, b]$ is a geodesic subsegment of $[1, x]$, we obtain

$$
\widehat{d}(r, b) \geqslant \frac{1}{2} \widehat{d}(1, x)-\frac{1}{2} \widehat{d}(1, p)-\frac{1}{2} \widehat{d}(b, x)-36 \delta-K_{1} .
$$

The points $r$ and $b$ lie on the geodesic $[1, x]$, and $\widehat{d}(b, x)=\widehat{d}(1, p)+K_{7}$ from $x$, so this implies

$$
\widehat{d}(r, x) \geqslant \frac{1}{2} \widehat{d}(1, x)+\frac{1}{2} K_{7}-36 \delta-K_{1} .
$$

Comparing (3.11) with (3.12) shows that the distance between the projections of the two halfspaces to $[1, x]$ is at least $7 \delta$, as $K_{7} \geqslant 92 \delta+2 K_{1}$. Therefore the nearest point projections of $H(1, x)$ and $H(b, a)$ are sufficiently far apart, and so $H(1, x) \subset H(a, b)$, as required.

We now prove the final statement, by an analogous argument to the one above, though unfortunately not exactly the same, as the picture is not completely symmetric.

Claim 3.12. For any point $b$ in $H(1, x)$, there is a point $a$ in $H(y, 1)$ with $\widehat{d}(a, b) \geqslant$ $2 A$, and $H(y, 1) \subset H(b, a)$.

Proof. Let $b$ be a point in the halfspace $H(1, x)$, and let $p$ be a nearest point projection of $b$ to $[1, x]$. Let $a$ be a point on the geodesic $[1, x]$, a distance $\widehat{d}(p, x)-$ $2 A-K_{7}$ from 1 , and this is greater than zero, as $\widehat{d}(1, x) \geqslant 4 A+2 K_{7}+6 \delta$. This is illustrated in Figure 11. 


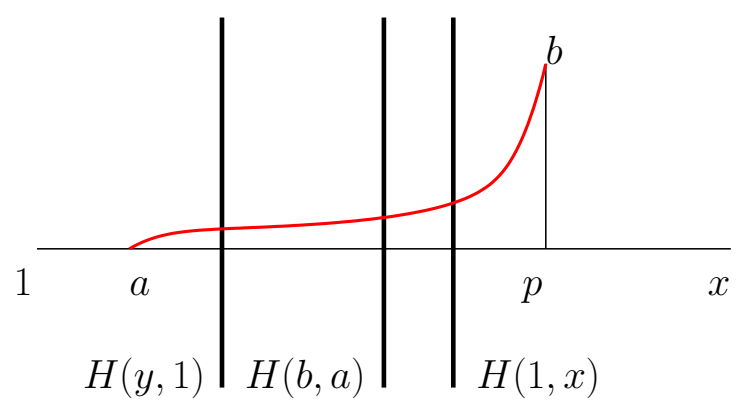

Figure 11 . The halfspace $H(y, 1)$ is contained in the halfspace $H(b, a)$.

We start by showing that $\widehat{d}(a, b) \geqslant 2 A$. Using Proposition 3.2 which says that the path $[a, p] \cup[p, b]$ is contained in a $3 \delta$-neighbourhood of $[a, b]$, and the triangle inequality, we obtain

$$
\widehat{d}(a, b) \geqslant \widehat{d}(a, p)-6 \delta .
$$

Proposition 3.7. $\widehat{d}(p, x) \leqslant \frac{1}{2} \widehat{d}(1, x)+3 \delta$, and our choice of $a$ imply that $\widehat{d}(1, a) \leqslant$ $\frac{1}{2} \widehat{d}(1, x)+3 \delta-2 A-K_{7}$. Therefore $\widehat{d}(a, p) \geqslant 2 A+K_{7}-6 \delta$, and so $\widehat{d}(a, b) \geqslant 2 A$ as $K_{7} \geqslant 12 \delta$.

We wish to show that $H(y, 1) \subset H(b, a)$, and as before it suffices to show that the nearest point projections of the halfspaces $H(y, 1)$ and $H(a, b)$ to the geodesic $[1, x]$ are at a distance at least $7 \delta$ apart, as the nearest point projection is coarsely well defined, Proposition 3.3. Let $r$ be a point in the nearest point projection of $H(y, 1)$ to $[1, x]$ which is furthest from 1. By Proposition 3.7, $\widehat{d}(1, r) \leqslant \frac{1}{2} \widehat{d}(1, y)+3 \delta$. Also, as $\widehat{d}(H(y, 1), H(1, x)) \geqslant A+K_{7}$, again using Proposition 3.7, this implies $\widehat{d}(x, y) \geqslant 2 A+2 K_{7}+6 \delta$. Therefore

$$
\widehat{d}(1, r) \leqslant \frac{1}{2} \widehat{d}(1, x)-A-K_{7}+6 \delta .
$$

Now applying Proposition 3.7 to the halfspace $H(a, b)$ implies that if $r$ is a point in the nearest point projection of $H(a, b)$ to $[a, b]$, then

$$
\widehat{d}(a, r) \geqslant \frac{1}{2} \widehat{d}(a, b)-3 \delta .
$$

Let $s$ be a closest point on $[a, b]$ to $p$. As $[a, s]$ is a subgeodesic of $[a, b]$, we may use Proposition 3.5 which implies that if $r$ is a point in the nearest point projection of $H(a, b)$ to $[a, s]$, then

$$
\widehat{d}(a, r) \geqslant \frac{1}{2} \widehat{d}(a, b)-3 \delta-K_{1} .
$$

By Proposition $3.2 \widehat{d}(s, p) \leqslant 3 \delta$, and the path $[a, p] \cup[p, s]$ is contained in a $3 \delta$ neighbourhood of $[a, s]$. Therefore, as the nearest point projections to close paths are close, Proposition 3.6, this implies that if $r$ is a nearest point in $[a, p] \cup[p, s]$ to $H(a, b)$, then

$$
\widehat{d}(a, r) \geqslant \frac{1}{2} \widehat{d}(a, b)-18 \delta-K_{1} .
$$

The nearest point projection onto the path $[a, p] \cup[p, s]$ is the same as the nearest point projection onto the path $[a, p] \cup[p, s] \cup[s, p]$, and this latter path is contained 
in a $3 \delta$-neighbourhood of $[a, p]$, so again applying Proposition 3.6, if $r$ lies in the nearest point projection of $H(a, b)$ to $[a, p]$, then

$$
\widehat{d}(a, r) \geqslant \frac{1}{2} \widehat{d}(a, b)-33 \delta-K_{1} \text {. }
$$

Using (3.13), and the fact that $[a, p]$ is a geodesic subsegment of $[1, x]$, we obtain

$$
\widehat{d}(1, r) \geqslant \frac{1}{2} \widehat{d}(1, p)+\frac{1}{2} \widehat{d}(1, a)-36 \delta-K_{1} .
$$

As we chose $a$ such that $\widehat{d}(1, a)=\widehat{d}(p, x)-2 A-K_{7}$, this shows

$$
\widehat{d}(1, r) \geqslant \frac{1}{2} \widehat{d}(1, x)-A-\frac{1}{2} K_{7}-36 \delta-K_{1} .
$$

Comparing (3.15) with (3.14) shows that the distance between the projections of the two halfspaces to $[1, x]$ is at least $7 \delta$, as $K_{7} \geqslant 98 \delta+2 K_{1}$. Therefore the nearest point projections of $H(y, 1)$ and $H(a, b)$ are sufficiently far apart, and so $H(y, 1) \subset H(b, a)$, as required.

This completes the proof of Proposition 3.10.

Finally, we prove a result about a slightly more general definition of halfspaces. Let $H(1, x ; C)=\{y \mid \widehat{d}(y, x) \leqslant \widehat{d}(1, x)+C\}$. We allow $C$ to be negative, and if $C$ is zero, this recovers the standard definition of a halfspace, and we shall continue to write $H(1, x)$ to mean $H(1, x ; 0)$. We now show that a coarse halfspace is contained in a halfspace.

Proposition 3.13. The halfspace $H(1, x ; C)$ is contained in the halfspace $H(1, y)$, for $\widehat{d}(1, y)=\widehat{d}(1, x)-C-9 \delta$ if $0 \leqslant C \leqslant \widehat{d}(1, x)-9 \delta$.

Proof. Let $z \in H(1, x ; C)$, and let $p$ be a nearest point to $z$ in $[1, x]$, so $\widehat{d}(z, x) \leqslant$ $\widehat{d}(z, 1)+C$. By the triangle inequality, $\widehat{d}(1, z) \leqslant \widehat{d}(1, p)+\widehat{d}(p, z)$; therefore

$$
\widehat{d}(z, x) \leqslant \widehat{d}(1, p)+\widehat{d}(p, z)+C
$$

The path from $z$ to $x$ via $p$ is almost a geodesic, Proposition 3.2 so $\widehat{d}(z, x) \geqslant$ $\widehat{d}(z, p)+\widehat{d}(p, x)-6 \delta$. This implies

$$
\widehat{d}(p, x) \leqslant \widehat{d}(1, p)+C+6 \delta .
$$

As $p$ lies on the geodesic $[1, x]$, we may rewrite this as

$$
\widehat{d}(p, x) \leqslant \frac{1}{2} \widehat{d}(1, x)+\frac{1}{2} C+3 \delta .
$$

By Proposition 3.7, this implies that $z$ is contained in the halfspace $H(1, y)$, for $\widehat{d}(1, y)=\widehat{d}(1, x)-C-9 \delta$, as required.

\section{NON-ELEMENTARY SEMI-GROUPS}

We do not assume that our random walk is symmetric, so it will be convenient to know that if the group generated by the support of $\mu$ is non-elementary, then the semi-group generated by the support of $\mu$ contains a pair of independent pseudo-Anosov elements. This follows from well-known results on the structure of subgroups of the mapping class group, which we now briefly review. We will use the definitions and results of Ivanov [12, although the results we obtain could also be deduced from work of McCarthy [30] and Birman, Lubotzky and McCarthy [1. 
We say an element $h$ of the mapping class group is pure if there is a disjoint collection of simple closed curves $\sigma(h)$ which are fixed individually by $h$, such that each complementary component of $\sigma(h)$ is also preserved, and furthermore $h$ acts on each complementary component as either a pseudo-Anosov element or the identity. If the collection of simple closed curves $\sigma(h)$ has the property that no simple closed curve with non-zero intersection number with $\sigma(h)$ is fixed by $h$, then $\sigma(h)$ is called a canonical reduction system for $h$. If $h$ is not pure, then we define the canonical reduction set $\sigma(h)$ to be the canonical reduction set of some pure power of $h$. Note that $\sigma\left(f g f^{-1}\right)=f \sigma(g)$. The canonical reduction system of a periodic or pseudoAnosov element of the mapping class group is empty. Ivanov shows that given reducible elements of the mapping class groups $f$ and $g$, there is a product of $f$ and $g$ whose canonical reduction set is the intersection of the canonical reduction sets of $f$ and $g$.

Lemma 4.1 ([12, Lemma 5.1]). Let $f$ and $g$ be reducible elements of the mapping class group. Then there are positive numbers $k$ and $l$ such that the canonical reduction set of $f^{k} g^{l}$ is the intersection of the canonical reduction sets for $f$ and $g$.

A pseudo-Anosov element $f$ acts on $\mathcal{P} \mathcal{M F}$ with simple dynamics, and there is a pair of fixed points, which we shall refer to as the stable fixed point $\lambda_{f}^{+}$and the unstable fixed point $\lambda_{f}^{-}$. The stable fixed point is attracting, i.e. every point in $\mathcal{P} \mathcal{M F} \backslash \lambda_{f}^{-}$converges to $\lambda_{f}^{+}$under iteration by $f$, and the unstable fixed point is repelling, i.e. every point in $\mathcal{P} \mathcal{M F} \backslash \lambda_{f}^{+}$converges to $\lambda_{f}^{-}$under iteration by $f^{-1}$. Ivanov [12] shows that a pair of pseudo-Anosov elements either has the same pair of fixed points or has disjoint fixed points.

Lemma 4.2 ([12, Lemma 5.11]). If $f$ and $g$ are pseudo-Anosov elements, then either $f i x(f)=f i x(g)$, or $f i x(f) \cap f i x(g)$ is empty.

We will also make use of the following observation from Ivanov [12].

Lemma 4.3 ([12, Chapter 11, exercise 3(a)]). Let $f$ be a pseudo-Anosov element, with fixed points $\lambda_{f}^{ \pm}$. Let $g$ be any element such that $g\left(\lambda_{f}^{+}\right) \neq \lambda_{f}^{-}$. Then $f^{n} g$ is pseudo-Anosov for all sufficiently large $n$.

We now show that if a non-elementary subgroup $M$ is generated by a semi-group $M^{+}$, then we can find a pair of independent pseudo-Anosov elements in $M^{+}$.

Lemma 4.4. Let $M^{+}$be a semi-group in the mapping class group which generates a non-elementary group $M$. Then $M^{+}$contains a pair of independent pseudo-Anosov elements.

Proof. The group $M$ contains two independent pseudo-Anosov elements. We now show that we can find a pair of independent pseudo-Anosov elements which in fact lie in $M^{+}$.

Suppose that $M^{+}$does not contain a pair of independent pseudo-Anosov elements, but does contain at least one pseudo-Anosov element. By Lemma 4.2, if $M^{+}$contains pseudo-Anosov elements, they must all have common endpoints. Let 
$f$ be a pseudo-Anosov element of $M^{+}$, with fixed points $\lambda_{f}^{+}$and $\lambda_{f}^{-}$. We will now show that all periodic and reducible elements of $M^{+}$preserve the fixed point of $f$.

Suppose $g$ is a periodic element of $M^{+}$. If $g$ does not preserve the fixed points of $f$, then $\mathrm{gfg}^{-1}$ is a pseudo-Anosov element with distinct fixed points, and furthermore lies in $M^{+}$, as $g^{-1}$ may be written as a positive power of $g$. So all periodic elements of $M^{+}$preserve the fixed points of $f$.

Suppose $g$ is a reducible element of $M^{+}$. At least one of $g$ or $g^{2}$ has the property that the image of $\lambda_{f}^{+}$is not $\lambda_{f}^{-}$, so possibly after replacing $g$ by its square, we may assume that $g$ has this property. Then by Lemma 4.3 the element $f^{n} g$ is pseudoAnosov for all sufficiently large $n$. In particular, $g f^{n} g g^{-1}=g f^{n}$ is pseudo-Anosov and lies in $M^{+}$. As we have assumed that $M^{+}$contains no independent pseudoAnosov elements, $g f^{n}$ must have the same fixed points as $f$, but this implies that $g$ preserves the fixed points of $f$.

We have shown that if $M^{+}$contains pseudo-Anosov elements, then they must all have common fixed points, and all other elements of $M^{+}$preserve the set of fixed points. But then the inverses of all elements of $M^{+}$preserve the set of fixed points, so in fact the entire group $M$ preserves the pair of fixed points, which contradicts the fact that $M$ is non-elementary. So we may assume that the semi-group $M^{+}$ contains no pseudo-Anosov elements.

If there is a disjoint collection of essential simple closed curves in the surface $\Sigma$ fixed by all elements of $M^{+}$, then this disjoint collection of simple closed curves is also fixed by all inverses of elements in $M^{+}$, and hence by the whole group $M$. As $M$ is non-elementary it fixes no disjoint collection of simple closed curves in $\Sigma$, so this implies that the semi-group $M^{+}$also fixes no disjoint collection of simple closed curves in the surface $\Sigma$. However, it could a priori be the case that all reducible elements of $M^{+}$have common simple closed curves in their canonical reduction sets, but these are not preserved by elliptic elements of $M^{+}$.

Let $\rho$ be the intersection of the canonical reduction sets of all reducible elements of $M^{+}$, i.e. $\rho=\bigcap\left\{\sigma(g) \mid g \in M^{+}, g\right.$ reducible $\}$. Lemma 4.1 shows that given reducible elements of the mapping class groups $f$ and $g$, there is an element of the semi-group generated by $f$ and $g$ whose canonical reduction set is the intersection of the canonical reduction sets for $f$ and $g$. This implies that there is a reducible element $r \in M^{+}$with $\sigma(r)=\rho$.

Suppose $g \in M^{+}$does not preserve $\rho$. Let $k$ be the smallest positive integer such that $g^{k}$ is pure. If $g$ is periodic, then $k$ is the order of $g$, and $\sigma(g)$ is empty. If $g$ is reducible, then $\rho \subset \sigma(g)$. By Lemma 4.3, for all sufficiently large positive integers $l$, the element $r^{l} g^{k}$ is pseudo-Anosov on each component of $\Sigma \backslash \rho$ on which either $r$ or $g^{k}$ is pseudo-Anosov. In particular, $\sigma\left(r^{l} g^{k}\right)=\rho$, and $r^{l} g^{k}$ is not periodic. As we have assumed $M^{+}$contains no pseudo-Anosov elements, $r^{l} g^{k}$ must in fact be reducible.

Consider conjugating $r^{l} g^{k}$ by $g$, i.e. $g f^{l} g^{k} g^{-1}=g f^{l} g^{k-1}$. This gives a reducible element of $M^{+}$with canonical reduction set $\sigma\left(g f^{l} g^{k-1}\right)=g \sigma\left(f^{l} g^{k}\right)=g(\rho) \neq \rho$. So we can construct an element of $M^{+}$with canonical reduction set $\rho \cap g(\rho)$, which is strictly smaller than $\rho$, which contradicts the fact that $\rho$ is the intersection of all of the canonical reduction sets of all of the reducible elements of $M^{+}$. So $\rho$ is preserved by all elements of $M^{+}$, and hence by the inverse of all elements of $M^{+}$, and therefore by the entire group $M$. But then the group $M$ is reducible, not non-elementary, a contradiction. 


\section{EXPONENTIAL DECAY}

In this section we show that the harmonic measure of halfspaces $H(1, x)$ decays exponentially with distance from the origin. As the number of disjoint halfspaces $H(1, x)$ grows exponentially in $\widehat{d}(1, x)$, this is clearly true on average, but in this section we show that the measure of all halfspaces decays as $L^{\widehat{d}(1, x)}$, for some constant $L<1$ which does not depend on the choice of halfspaces. We then show that there is a similar estimate for the convolutions measures $\mu_{n}$, i.e. $\mu_{n}(H(1, x)) \leqslant$ $Q L^{\widehat{d}(1, x)}$, for some constant $Q$, and where $L<1$ is the same constant as for $\nu$.

We will need to use the fact that the group generated by the support of the random walk is non-elementary, and it will be convenient to use the fact that nonelementary subgroups have free subgroups that act on the complex of curves in a similar manner to Schottky groups acting on hyperbolic space.

Definition 5.1. We say that a pair of elements $a, b \in G$ is a Schottky pair if there are disjoint halfspaces $A^{+}, A^{-}, B^{+}$and $B^{-}$in the relative space $\widehat{G}$, such that $a\left(\widehat{G} \backslash A^{-}\right) \subset A^{+}, a^{-1}\left(\widehat{G} \backslash A^{+}\right) \subset A^{-}, b\left(\widehat{G} \backslash B^{-}\right) \subset B^{+}$and $b^{-1}\left(\widehat{G} \backslash B^{+}\right) \subset B^{-}$. We will refer to a choice of halfspaces $A^{ \pm}, B^{ \pm}$, with the properties above, as Schottky halfspaces for the Schottky pair $a, b$.

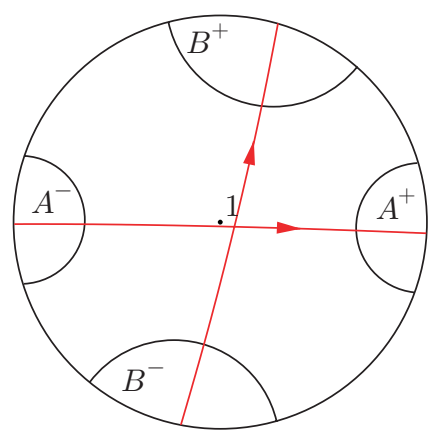

FiguRE 12. Halfspaces for a Schottky pair.

This is illustrated in Figure 12, The red lines (gray) correspond to axes for $a$ and $b$. The subgroup generated by a Schottky pair is a free group with two generators, in which all non-identity elements are pseudo-Anosov. A non-elementary subgroup of the mapping class group contains a pair of pseudo-Anosov elements with distinct endpoints in $\mathcal{P} \mathcal{M F}$, and these correspond to distinct points in the Gromov boundary of the complex of curves by work of Klarreich 21]. Therefore there are integers $p$ and $q$ such that $a^{p}$ and $b^{q}$ are a Schottky pair. Furthermore, we may replace $p$ and $q$ with any larger integers.

We first show that the harmonic measure of a Schottky halfspace is non-zero, for any Schottky pair contained in the support of $\mu$.

Proposition 5.2. Let $A^{+}$be a Schottky halfspace, whose corresponding pseudoAnosov element lies in the subgroup generated by the support of $\mu$. Then $\nu\left(\overline{A^{+}}\right)>0$.

Proof. Suppose $\nu\left(\overline{A^{+}}\right)=0$. The measure $\nu$ is $\mu_{n}$-invariant, i.e. $\nu(X)=$ $\sum \mu_{n}(g) \nu\left(g^{-1} X\right)$, so if $\mu_{n}(g) \neq 0$, then $\nu\left(g^{-1} \overline{A^{+}}\right)=0$. As $a$ lies in the support of $\mu$, there is an $n$ such that $\mu_{n}(a)>0$. This implies that $\nu\left(a^{-k} \overline{A^{+}}\right)=0$ for 
all $k$. However, the complement of the union of the $a^{-k} A^{+}$is the unstable fixed point of $a$, which has measure zero as $\nu$ is non-atomic. This means that the total measure $\nu(\mathcal{P} \mathcal{M F})$ is zero, a contradiction.

We now show that the harmonic measure of $H(1, x)$ is bounded away from 1 , at least for $\widehat{d}(1, x)$ sufficiently large.

Proposition 5.3. Let $\mu$ be a probability distribution on $G$ whose support generates a non-elementary subgroup, and let $\nu$ be the corresponding harmonic measure. Then there are constants $K_{8}$ and $\epsilon>0$ such that $\nu(\overline{H(1, x)}) \leqslant 1-\epsilon$, for all $x$ with $\widehat{d}(1, x) \geqslant K_{8}$. The constant $K_{8}$ only depends on the support of $\mu$. The constant $\epsilon$ depends on $\mu$.

Proof. Let $M$ be the subgroup generated by the support of $\mu$. As this subgroup is non-elementary, it contains a Schottky pair, $\{a, b\}$. As $a$ and $b$ are pseudoAnosov, the sequences $a^{k}$ and $b^{k}$ are quasi-geodesic, with distinct endpoints, so possibly after replacing $a$ and $b$ by positive powers $a^{r}$ and $b^{s}$, we may assume that $\left\{H\left(1, a^{ \pm 1}\right), H\left(1, b^{ \pm 1}\right)\right\}$ is a collection of Schottky halfspaces for $\{a, b\}$, and $\min \{\widehat{d}(1, a), \widehat{d}(1, b)\}>2 \widehat{d}(1,[a, b])+K_{4}$. By Proposition 3.9 there is a $K_{8}=$ $2 \widehat{d}(1,[a, b])+K_{5}$ such that any halfspace $H(1, x)$ with $\widehat{d}(1, x) \geqslant K_{8}$ intersects at most one of the Schottky halfspaces. In particular this means that the halfspace $H(1, x)$ is disjoint from at least three of the Schottky halfspaces, so we may choose $\epsilon$ to be the minimum of the sum of any three of the harmonic measures of the four Schottky halfspaces.

We now show that the harmonic measure of halfspaces $H(1, x)$ decays exponentially in $\widehat{d}(1, x)$ as $x$ varies, at least for $x$ with $\widehat{d}(1, x)$ sufficiently large.

Lemma 5.4. Let $\mu$ be a probability distribution on $G$ whose support is relatively bounded and which generates a non-elementary subgroup, and let $\nu$ be the corresponding harmonic measure. Then there are constants $K_{9}$ and $L<1$, such that if $\widehat{d}(1, x) \geqslant K_{9}$, then $\nu(\overline{H(1, x)}) \leqslant L^{\widehat{d}(1, x)}$. Both $K_{9}$ and $L$ depend on $\mu$.

Proof. As the subgroup generated by the support of $\mu$ is non-elementary, the subgroup contains a Schottky pair, so by Proposition 5.3. there are constants $K_{8}$ and $\epsilon$ such that $\nu(\overline{H(1, x)}) \leqslant 1-\epsilon$, for $\widehat{d}(1, x) \geqslant K_{8}$.

Before considering more general random walks, we explain the argument in the case of the nearest neighbour random walk on the Cayley graph. Consider a pair of nested halfspaces $H\left(1, x_{2}\right)$ and $H\left(1, x_{2}\right)$, where $\widehat{d}\left(1, x_{2}\right) \geqslant 2 K_{8}+K_{6}$, and $x_{1} \in\left[1, x_{2}\right]$ with $\widehat{d}\left(1, x_{1}\right)=\widehat{d}\left(1, x_{2}\right)-K_{8}-2 K_{7}$. Given a halfspace $H\left(1, x_{1}\right)$, we will refer to the set of points which are an equal distance from both 1 and $x_{1}$ as the equidistant set for $H\left(1, x_{1}\right)$, which we shall denote by $E\left(1, x_{1}\right)$. We remark that a priori it may be the case that the equidistant set $E\left(1, x_{1}\right)=H\left(1, x_{1}\right) \cap H\left(x_{1}, 1\right)$ has measure strictly larger than zero. Any sample path that converges into the limit set of $H\left(1, x_{2}\right)$ must pass through the equidistant set of $H\left(1, x_{1}\right)$. So we can use the formula for conditional probability, conditioning on the location at which the sample path first hits the equidistant set of $H\left(1, x_{1}\right)$. By Proposition 3.10, for any point $a \in E\left(1, x_{1}\right)$, the halfspace $H\left(1, x_{2}\right)$ is contained in a halfspace $H(a, b)$, with $\widehat{d}(a, b) \geqslant K_{8}$, so the probability that you converge into the limit set of $H\left(1, x_{2}\right)$ from any point of $E\left(1, x_{1}\right)$ is at most $1-\epsilon$, so, in particular, the probability that you converge into the limit set of $H\left(1, x_{2}\right)$ is at most $1-\epsilon$. We may apply this 
argument to any nested sequence of halfspaces, each a distance at least $2 K_{8}+K_{6}$ apart, so the probability that you converge into the limit set of a halfspace $H(1, x)$ with $\widehat{d}(1, x) \geqslant\left(2 K_{8}+K_{6}\right) n$ is at most $(1-\epsilon)^{(n-1)}$.

For more general probability distributions, we need to replace the condition of hitting the equidistant set of a halfspace, with the condition of hitting some suitably large neighbourhood of the equidistant set.

Let $H_{i}$ be a sequence of halfspaces $H\left(1, x_{i}\right)$, where the $x_{i}$ lie on a common geodesic starting at 1 , with $\widehat{d}\left(1, x_{i+1}\right) \geqslant \widehat{d}\left(1, x_{i}\right)+2 K_{8}+K_{6}$ for each $i$. Consider a sample path which converges to a boundary point $\lambda$ in $\bar{H}_{k}$. Such a sample path must have infinitely many elements in $H_{1}$. If such a sample path does not hit $H_{1} \backslash H_{k}$, then it must first hit $H_{1}$ inside $H_{k}$, after a jump of distance at least $k\left(2 K_{8}+K_{6}\right)$. Pick $k$ such that $k\left(2 K_{8}+K_{6}\right)$ is larger than the relative diameter of the support of $\mu$.

For a sample path $w$, let $F(w)$ be the group element corresponding to the location at which $\omega$ first hits $H_{1}$. This is well defined for sample paths which converge to $\lambda \in \bar{H}_{2}$. All sample paths which converge to $\lambda \in \bar{H}_{k}$ hit $H_{1} \backslash H_{k}$, so conditioning on $F(\omega)$ gives

$$
\mathbb{P}\left(\lambda(\omega) \in \bar{H}_{k+1} \mid \lambda(\omega) \in \bar{H}_{k}\right) \leqslant \sum_{g \in H_{1} \backslash H_{k}} \mathbb{P}(F(\omega)=g) \nu\left(g^{-1} \overline{H_{k+1}}\right) .
$$

By Proposition 3.10, for any point $g$ in $H_{1} \backslash H_{k}$, there is a point $b$ in $H_{k+1}$ such that $H_{k+1} \subset H(g, b)$, and $\widehat{d}(g, b) \geqslant K_{8}$. Therefore, by Proposition $\left[5.3, \nu\left(g^{-1} \overline{H_{k+1}}\right) \leqslant\right.$ $1-\epsilon$, so

$$
\mathbb{P}\left(\lambda(\omega) \in \bar{H}_{k+1} \mid \lambda(\omega) \in \bar{H}_{k}\right) \leqslant(1-\epsilon) .
$$

This implies that $\nu\left(\overline{H_{k+1}}\right) \leqslant \nu\left(\overline{H_{k}}\right)(1-\epsilon)$, so by induction $\nu\left(\overline{H_{k+l}}\right) \leqslant(1-\epsilon)^{l+1}$. Therefore $\nu\left(\overline{H_{n}}\right) \leqslant L^{n}$, for $\widehat{d}(1, x) \geqslant K_{9}$, where we may choose $L=(1-\epsilon)^{1 /(k+1)}$ and $K_{9}=k\left(2 K_{8}+K_{6}\right)$.

The measure $\nu$ is the weak limit of the measures $\mu_{n}$, so one may hope there is exponential decay for halfspaces for the $\mu_{n}$-measures, at least for large $n$. In fact, we now show that there is an upper bound for $\mu_{n}(H(1, x))$ which decays exponentially in $\widehat{d}(1, x)$, for all $n$.

Lemma 5.5. There are constants $K_{10}, L<1$ and $Q$, which only depend on $\delta$ and $\mu$, such that if $H(1, x)$ is a halfspace with $\widehat{d}(1, x) \geqslant K_{10}$, then $\mu_{n}(H(1, x)) \leqslant Q L^{\widehat{d}(1, x)}$.

Proof. We shall choose $K_{10}=K_{6}+2 K_{8}+K_{9}$. Let $H(1, x)$ be a halfspace with $\widehat{d}(1, x) \geqslant 2 K_{8}+K_{6}$. Then, by Proposition 3.10, we may choose $y \in[1, x]$ with $\widehat{d}(1, y)=\widehat{d}(1, x)-K_{8}-2 K_{7}$, such that $H(1, x) \subset H(1, y)$, and, furthermore, for any $b \in H(1, x)$, there is a point $a$ in $H(y, 1)$ such that the halfspace $H(y, 1)$ is contained in the halfspace $H(b, a)$, with $\widehat{d}(a, b) \geqslant K_{8}$. By Proposition [5.3 the harmonic measure of $\overline{H(y, 1)}$ viewed from any $b \in H(1, x)$ is at most $1-\epsilon$, i.e. if $\nu_{b}$ is the harmonic measure induced by a random walk starting at $b$ instead of 1 , then $\nu_{b}(\overline{H(y, 1)}) \leqslant 1-\epsilon$. At time $n$, the halfspace $H(1, x)$ has measure $\mu_{n}(H(1, x))$, so the proportion of these sample paths which converge into the limit set of $H(y, 1)$ is at most $1-\epsilon$. Therefore at least $\epsilon$ of these sample paths converge into the complement of the limit set of $H(y, 1)$, which is contained in the limit set 
of $H(1, y)$, i.e. $\nu(\overline{H(1, y)}) \geqslant \epsilon \mu_{n}(H(1, x))$. This implies

$$
\mu_{n}(H(1, x)) \leqslant \frac{1}{\epsilon} \nu(\overline{H(1, y)}) .
$$

We chose $\widehat{d}(1, x) \geqslant K_{6}+2 K_{8}+K_{9}$ and $\widehat{d}(1, y)=\widehat{d}(1, x)-K_{8}-2 K_{7}$, so we may apply the exponential decay bounds from Lemma 5.4 to obtain

$$
\mu_{n}(H(1, x)) \leqslant \frac{1}{\epsilon} L^{\widehat{d}(1, x)-K_{8}-2 K_{7}} .
$$

Therefore we may choose $Q$ to be $\frac{1}{\epsilon} L^{-K_{8}-2 K_{7}}$, and this depends on $\delta$ and $\mu$, but not on $x$ or $n$. Therefore we have shown that there is a constant $Q$ such that $\mu_{n}(H(1, x)) \leqslant Q L^{\widehat{d}(1, x)}$, for all $n$, as long as $\widehat{d}(1, x) \geqslant K_{10}=K_{6}+2 K_{8}+K_{9}$. The constant $L$ may be chosen to have the same value as the constant $L$ from Lemma 5.4 .

We remark that for the nearest neighbour random walk, for small $n$ and $\widehat{d}(1, x)$ large, $\mu_{n}(H(1, x))$ will be zero until $n$ is at least $\widehat{d}(1, x)$, so $\mu_{n}(H(1, x))$ need not be monotonically decreasing in $n$.

\section{LiNEAR PROGRESS}

We may now complete the proof of the main theorem.

Theorem 1.1. Let $G$ be the mapping class group of an orientable surface of finite type, which is not a sphere with three or fewer punctures, and consider the random walk generated by a probability distribution $\mu$, whose support is bounded in the relative metric, which generates a non-elementary subgroup of the mapping class group, and which has finite first moment. Then there is a constant $\ell>0$ such that $\lim _{n \rightarrow \infty} \frac{1}{n} \widehat{d}\left(1, w_{n}\right)=\ell$ almost surely.

The fact that the limit $\frac{1}{n} \widehat{d}\left(1, w_{n}\right)$ exists almost surely with respect to $\mathbb{P}$ follows from a standard application of Kingman's subadditive ergodic theorem. The main task of this section is to show that this limit is strictly greater than zero. We now state Kingman's subadditive ergodic theorem 20, using the version from Woess [35, Theorem 8.10].

Theorem $6.1(20,35)$. Let $(\Omega, \mathbb{P})$ be a probability space and $U: \Omega \rightarrow \Omega$ a measurepreserving transformation. If $W_{n}$ is a subadditive sequence of non-negative realvalued random variables on $\Omega$, that is, $W_{n+k} \leqslant W_{n}+W_{k} \circ U^{n}$ for all $k, n \in \mathbb{N}$, and $W_{1}$ has finite first moment, then there is a $U$-invariant random variable $W_{\infty}$ such that

$$
\lim _{n \rightarrow \infty} \frac{1}{n} W_{n}=W_{\infty}
$$

$\mathbb{P}$-almost surely, and in expectation.

We will choose $(\Omega, \mathbb{P})$ to be the path space $\left(G^{\mathbb{Z}_{+}}, \mathbb{P}\right)$ for the random walk determined by $(G, \mu)$. We will choose $U$ to be the Bernoulli shift in the space of increments of the random walk, which is an ergodic measure-preserving transformation on $\left(G^{\mathbb{Z}_{+}}, \mathbb{P}\right)$. Set $W_{n}=\widehat{d}\left(1, w_{n}\right)$. Then $W_{k} \circ U^{n}=\widehat{d}\left(1, w_{n}^{-1} w_{n+k}\right)$, which is the relative distance between $w_{n}$ and $w_{n+k}$, so the triangle inequality implies that $W_{n}$ is subadditive. We have assumed that $\mu$ has finite first moment with respect to the word metric on $G$, and as $\widehat{d}(1, g) \leqslant|g|$ for all $g \in G$, this implies that $\mu$ also has finite first moment with respect to the relative metric on $G$. Theorem 6.1 
then implies that $\lim _{n \rightarrow \infty} \frac{1}{n} \widehat{d}\left(1, w_{n}\right)=\ell$ exists almost surely, and in expectation, and in fact is constant almost surely. As $\widehat{d}\left(1, w_{n}\right) \geqslant 0$ for all $n$, this implies that $\ell \geqslant 0$. It remains to show that $\ell$ is strictly greater than zero. As the limit is constant almost surely, and the limit exists in expectation, it suffices to show that the limit $\frac{1}{n} \mathbb{E}\left(\widehat{d}\left(1, w_{n}\right)\right)$ is bounded away from zero. We will show that for $m$ sufficiently large, the expected difference between $\widehat{d}\left(1, w_{n}\right)$ and $\widehat{d}\left(1, w_{n+m}\right)$ is bounded away from zero.

Lemma 6.2. There is a constant $N$, such that if $m>N$, then $\mathbb{E}\left(\widehat{d}\left(1, w_{n+m}\right)-\right.$ $\left.\widehat{d}\left(1, w_{n}\right)\right) \geqslant \delta>0$. The constant $N$ depends on $\mu$, but is independent of $m$ and $n$.

This suffices to prove Theorem [1.1, as we now explain. Consider

$$
\mathbb{E}\left(\widehat{d}\left(1, w_{2 N}\right)\right)=\mathbb{E}\left(\widehat{d}\left(1, w_{2 N}\right)-\widehat{d}\left(1, w_{N}\right)+\widehat{d}\left(1, w_{N}\right)-\widehat{d}\left(1, w_{0}\right)\right) .
$$

This is equal to

$$
=\mathbb{E}\left(\widehat{d}\left(1, w_{2 N}\right)-\widehat{d}\left(1, w_{N}\right)\right)+\mathbb{E}\left(\widehat{d}\left(1, w_{N}\right)\right) \geqslant 2 \delta .
$$

A similar argument shows $\mathbb{E}\left(\widehat{d}\left(1, w_{k N}\right)\right) \geqslant k \delta$. As $\lim _{n \rightarrow \infty} \frac{1}{n} \widehat{d}\left(1, \omega_{n}\right)=\ell$ is constant almost surely, this shows $\ell \geqslant \delta / N>0$, as required.

We now prove Lemma 6.2.

Proof. Consider doing a random walk of length $n$, followed by one of length $m$. We can compute the expected change in relative length from time $n$ to time $n+m$, which we shall denote $\Delta_{n, m}$ :

$$
\Delta_{n, m}=\mathbb{E}\left(\widehat{d}\left(1, w_{n+m}\right)-\widehat{d}\left(1, w_{n}\right)\right) .
$$

We may rewrite this using the definition of expected value, giving

$$
\Delta_{n, m}=\sum_{x \in G} \mu_{n}(x) \sum_{y \in G} \mu_{m}(y)(\widehat{d}(1, x y)-\widehat{d}(1, x)) .
$$

The fact that $\mu$ has finite first moment means that this sum is absolutely convergent, so we can swap the order of summation. Furthermore, as $\widehat{d}(1, x y)=\widehat{d}\left(x^{-1}, y\right)$ and $\widehat{d}(1, x)=\widehat{d}\left(x^{-1}, 1\right)$, we can rewrite this as

$$
\Delta_{n, m}=\sum_{y \in G} \mu_{m}(y) \sum_{x \in G} \mu_{n}(x)\left(\widehat{d}\left(x^{-1}, y\right)-\widehat{d}\left(x^{-1}, 1\right)\right) .
$$

We may split the sum up into two parts, depending on whether or not $\widehat{d}(1, y)>A$, where $A$ is a constant which depends on $\mu$. We will choose $A \geqslant K_{6}+2 K_{8}+K_{9}+12 \delta$, and furthermore, we will choose $A$ to be sufficiently large such that $r L^{r}<\delta / 2 Q$, for all real numbers $r \geqslant A$, and this is possible as $L<1$. Here the constants $K_{6}, K_{8}, K_{9}, L$ and $Q$ are those defined previously in Sections 3 and 5 though we emphasize that $Q$ and $L$ are the constants defined for the reflected random walk $(G, \widetilde{\mu})$. We shall write $\widehat{B}_{A}$ for the ball of radius $A$ about 1 in $\widehat{G}$. Then

$$
\begin{aligned}
\Delta_{n, m} & =\sum_{y \in \widehat{B}_{A}} \mu_{m}(y) \sum_{x \in G} \mu_{n}(x)\left(\widehat{d}\left(x^{-1}, y\right)-\widehat{d}\left(x^{-1}, 1\right)\right) \\
& +\sum_{y \in G \backslash \widehat{B}_{A}} \mu_{m}(y) \sum_{x \in G} \mu_{n}(x)\left(\widehat{d}\left(x^{-1}, y\right)-\widehat{d}\left(x^{-1}, 1\right)\right) .
\end{aligned}
$$


We now find lower bounds for lines (6.1) and (6.2) in turn. First, in line (6.1), $\widehat{d}\left(x^{-1}, y\right)-\widehat{d}\left(x^{-1}, 1\right) \geqslant-\widehat{d}(1, y)$, by the triangle inequality. We have also assumed that $\widehat{d}(1, y) \leqslant A$. This implies the following lower bound for (6.1):

$$
\text { (6.1) } \geqslant-\mu_{m}\left(\widehat{B}_{A}\right) A \text {. }
$$

We now find a lower bound for line (6.2), and we will simplify the notation by replacing $x$ with $x^{-1}$. This makes no difference to the sum as we are summing over all elements of $G$ :

$$
\left(\underline{6.2)} \geqslant \sum_{y \in G \backslash \widehat{B}_{A}} \mu_{m}(y) \sum_{x \in G} \mu_{n}\left(x^{-1}\right)(\widehat{d}(x, y)-\widehat{d}(x, 1)) .\right.
$$

Let $N$ be the collection of $x \in G$ for which $\widehat{d}(x, y)-\widehat{d}(x, 1) \leqslant 0$, i.e. $N$ is the halfspace $H(1, y)$. Let $P$ be the region on which $\widehat{d}(x, y)-\widehat{d}(x, 1) \geqslant 3 \delta$, so $P$ is the halfspace $H(y, 1 ;-3 \delta)$, using the notation from Proposition 3.13. This is illustrated in Figure 13

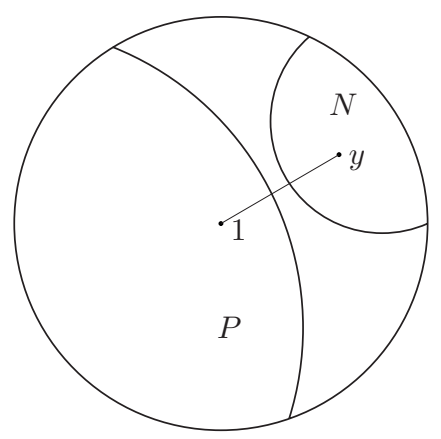

Figure 13. Positive and negative regions.

Let $z$ be a point on $[1, y]$ at a distance $12 \delta$ from $y$. We now show that the region $P$ contains the halfspace $H(z, 1)$. The complement of $P$ is contained in $H(1, y ; 3 \delta)$, and by Proposition 3.13 , the complement of $P$ is contained in $H(1, z)$, for $\widehat{d}(1, z)=\widehat{d}(1, y)-12 \delta$. Therefore $P$ contains the halfspace $H(z, 1)$. By the triangle inequality, $\widehat{d}(x, y)-\widehat{d}(x, 1) \geqslant-\widehat{d}(1, y)$ on $N$, and $\widehat{d}(x, y)-\widehat{d}(x, 1) \geqslant 3 \delta$ on $P$. This gives the following lower bound for line (6.2):

$$
\left(\underline{6.2)} \geqslant \sum_{y \in G \backslash \widehat{B}_{A}} \mu_{m}(y)\left[-\widehat{d}(1, y) \widetilde{\mu}_{n}(H(1, y))+3 \delta \widetilde{\mu}_{n}(H(z, 1))\right] .\right.
$$

Here $\widetilde{\mu}_{n}$ is the reflected random walk measure, i.e. $\widetilde{\mu}_{n}(x)=\mu_{n}\left(x^{-1}\right)$. Using the fact that $\widetilde{\mu}_{n}(H(z, 1)) \geqslant 1-\widetilde{\mu}_{n}(H(1, z))$, we obtain

$$
\left(\underline{6.2)} \geqslant \sum_{y \in G \backslash \widehat{B}_{A}} \mu_{m}(y)\left[-\widehat{d}(1, y) \widetilde{\mu}_{n}(H(1, y))+3 \delta\left(1-\widetilde{\mu}_{n}(H(1, z))\right)\right] .\right.
$$


In line (6.2) we have assumed that $\widehat{d}(1, y) \geqslant 2 K_{8}+K_{6}+12 \delta$, so the halfspaces $H(1, y)$ and $H(1, z)$ both satisfy the hypotheses of Lemma 5.5, so we may estimate $\widetilde{\mu}_{n}$ in terms of $\widetilde{\nu}$ :

$$
\left(\underline{6.2)} \geqslant \sum_{y \in G \backslash \widehat{B}_{A}} \mu_{m}(y)[3 \delta-\widehat{d}(1, y) Q \widetilde{\nu}(\overline{H(1, y)})-\delta Q \widetilde{\nu}(\overline{H(z, 1)})] .\right.
$$

Here $\widetilde{\nu}$ is the harmonic measure corresponding to the reflected random walk defined by $(G, \widetilde{\mu})$, and $Q$ is a constant which depends on $\widetilde{\mu}$, but not on $y$ or $z$. In line (6.2) we have assumed that $\widehat{d}(1, y) \geqslant K_{9}+12 \delta$, so we may apply Lemma 5.4 . which says that the harmonic measure of halfspaces decays exponentially:

$$
(\underline{6.2}) \geqslant \sum_{y \in G \backslash \widehat{B}_{A}} \mu_{m}(y)\left[3 \delta-Q \widehat{d}(1, y) L^{\widehat{d}(1, y)}-Q \delta L^{\widehat{d}(1, z)}\right] .
$$

Now using the fact that $\widehat{d}(1, y) \geqslant \delta$, and we chose $A$ such that $r L^{r} \leqslant \delta / 2 Q$ for all $r \geqslant A$, this implies that the term in square brackets in the line above is at least $2 \delta$. Therefore

$$
\text { (6.2) } \geqslant\left(1-\mu_{m}\left(\widehat{B}_{A}\right)\right) 2 \delta .
$$

Recall that $\Delta_{n, m}=6$ (6.1) +6.2 , and now using the lower bounds from lines (6.3) and (6.4), we obtain

$$
\Delta_{n, m} \geqslant 2 \delta-\mu_{m}\left(\widehat{B}_{A}\right)(A+2 \delta)
$$

The harmonic measure of a bounded set in $\widehat{G}$ is zero, and so the random walk is transient on relatively bounded sets. In particular, for fixed $A$ there is an $N$ such that $\mu_{m}\left(\widehat{B}_{A}\right)<\delta /(A+2 \delta)$ for all $m \geqslant N$, and this will be our choice of $N$, which depends on $\mu$. In particular, this means that $\Delta_{n, m} \geqslant \delta$, for all $n$, and for all $m \geqslant N$, and so $\Delta_{n, m}$ is bounded away from zero, as required.

This completes the proof of Lemma 6.2, and hence of Theorem 1.1 .

\section{ACKNOWLEDGEMENTS}

The author would like to thank the referee for many helpful comments. This work was partially supported by NSF grant DMS-0706764.

\section{REFERENCES}

[1] Joan S. Birman, Alex Lubotzky, and John McCarthy, Abelian and solvable subgroups of the mapping class groups, Duke Math. J. 50 (1983), no. 4, 1107-1120. MR.726319 (85k:20126)

[2] Brian H. Bowditch, Hyperbolic 3-manifolds and the geometry of the curve complex, European Congress of Mathematics, Eur. Math. Soc., Zürich, 2005, pp. 103-115. MR2185739 (2006h:57014)

[3] Martin R. Bridson and André Haefliger, Metric spaces of non-positive curvature, Grundlehren der Mathematischen Wissenschaften [Fundamental Principles of Mathematical Sciences], vol. 319, Springer-Verlag, Berlin, 1999. MR.1744486 (2000k:53038)

[4] Mahlon Marsh Day, Convolutions, means, and spectra, Illinois J. Math. 8 (1964), 100-111. MR0159230 (28:2447)

[5] M. Dehn, Die Gruppe der Abbildungsklassen, Acta Math. 69 (1938), no. 1, 135-206 (German). Das arithmetische Feld auf Flächen. MR 1555438

[6] Moon Duchin, Thin triangles and a multiplicative ergodic theorem for Teichmüller geometry, available at math.GT/0508046 
[7] B. Farb, Relatively hyperbolic groups, Geom. Funct. Anal. 8 (1998), no. 5, 810-840. MR 1650094 (99j:20043)

[8] Benson Farb and Howard Masur, Superrigidity and mapping class groups, Topology 37 (1998), no. 6, 1169-1176. MR1632912 (99f:57017)

[9] Ursula Hamenstädt, Train tracks and the Gromov boundary of the complex of curves, Spaces of Kleinian groups, London Math. Soc. Lecture Note Ser., vol. 329, Cambridge Univ. Press, Cambridge, 2006, pp. 187-207. MR 2258749

[10] Kevin Hartshorn, Heegaard splittings of Haken manifolds have bounded distance, Pacific J. Math. 204 (2002), no. 1, 61-75. MR1905192 (2003a:57037)

[11] John Hempel, 3-manifolds as viewed from the curve complex, Topology 40 (2001), no. 3, 631-657. MR1838999(2002f:57044)

[12] Nikolai V. Ivanov, Subgroups of Teichmüller modular groups, Translations of Mathematical Monographs, vol. 115, American Mathematical Society, Providence, RI, 1992. Translated from the Russian by E. J. F. Primrose and revised by the author. MR 1195787 (93k:57031)

[13] Vadim A. Kaimanovich, The Poisson formula for groups with hyperbolic properties, Ann. of Math. (2) 152 (2000), no. 3, 659-692. MR.1815698 (2002d:60064)

[14] Vadim A. Kaimanovich and Howard Masur, The Poisson boundary of the mapping class group, Invent. Math. 125 (1996), no. 2, 221-264. MR1395719 (97m:32033)

[15] Anders Karlsson, On the dynamics of isometries, Geom. Topol. 9 (2005), 2359-2394 (electronic). MR2209375 (2007h:22014)

[16] Anders Karlsson and Gregory A. Margulis, A multiplicative ergodic theorem and nonpositively curved spaces, Comm. Math. Phys. 208 (1999), no. 1, 107-123. MR.1729880(2000m:37031)

[17] Steven P. Kerckhoff, The measure of the limit set of the handlebody group, Topology 29 (1990), no. 1, 27-40. MR1046623 (91b:57011)

[18] Harry Kesten, Full Banach mean values on countable groups, Math. Scand. 7 (1959), 146-156. MR0112053 (22:2911)

[19] Symmetric random walks on groups, Trans. Amer. Math. Soc. 92 (1959), 336-354. MR0109367 (22:253)

[20] J. F. C. Kingman, The ergodic theory of subadditive stochastic processes, J. Roy. Statist. Soc. Ser. B 30 (1968), 499-510. MR0254907 (40:8114)

[21] E. Klarreich, The boundary at infinity of the curve complex and the relative Teichmüller space.

[22] Tsuyoshi Kobayashi, Casson-Gordon's rectangle condition of Heegaard diagrams and incompressible tori in 3-manifolds, Osaka J. Math. 25 (1988), no. 3, 553-573. MR969018 (89m:57016)

[23] Emmanuel Kowalski, The principle of the large sieve, available at arXiv:math.NT/0610021.

[24] W. B. R. Lickorish, A finite set of generators for the homeotopy group of a 2-manifold, Proc. Cambridge Philos. Soc. 60 (1964), 769-778. MR0171269 (30:1500)

[25] Joseph Maher, Random walks on the mapping class group, available at arXiv:math.GT/ 0604433

[26] Howard A. Masur and Yair N. Minsky, Quasiconvexity in the curve complex, In the tradition of Ahlfors and Bers, III, Contemp. Math., vol. 355, Amer. Math. Soc., Providence, RI, 2004, pp. 309-320. MR.2145071(2006a:57022)

[27] - Geometry of the complex of curves. I. Hyperbolicity, Invent. Math. 138 (1999), no. 1, 103-149. MR1714338 (2000i:57027)

[28] H. A. Masur and Y. N. Minsky, Geometry of the complex of curves. II. Hierarchical structure, Geom. Funct. Anal. 10 (2000), no. 4, 902-974. MR:1791145(2001k:57020)

[29] Howard A. Masur and Yair N. Minsky, Unstable quasi-geodesics in Teichmüller space, In the tradition of Ahlfors and Bers (Stony Brook, NY, 1998), Contemp. Math., vol. 256, Amer. Math. Soc., Providence, RI, 2000, pp. 239-241. MR1759683 (2001m:32031)

[30] John McCarthy, A "Tits-alternative" for subgroups of surface mapping class groups, Trans. Amer. Math. Soc. 291 (1985), no. 2, 583-612. MR800253 (87f:57011)

[31] Yair N. Minsky, Extremal length estimates and product regions in Teichmüller space, Duke Math. J. 83 (1996), no. 2, 249-286. MR.1390649 (97b:32019)

[32] R. C. Penner and J. L. Harer, Combinatorics of train tracks, Annals of Mathematics Studies, vol. 125, Princeton University Press, Princeton, NJ, 1992. MR.1144770 (94b:57018)

[33] Kasra Rafi and Saul Schleimer, Covers and the curve complex, Geom. Topol. 13 (2009), no. 4, 2141-2162. MR2507116 
[34] Igor Rivin, Walks on groups, counting reducible matrices, polynomials and surface and free group automorphisms, Duke Math. J. 142 (2008), no. 2, 353-379. MR2401624|(2009m:20077)

[35] Wolfgang Woess, Random walks on infinite graphs and groups, Cambridge Tracts in Mathematics, vol. 138, Cambridge University Press, Cambridge, 2000. MR.1743100 (2001k:60006)

Department of Mathematics, College of Staten Island (CUNy), Staten Island, New YORK 10314

E-mail address: joseph.maher@csi.cuny.edu 